Karl-Franzens Universität Graz

Technische Universität Graz

Medizinische Universität Graz

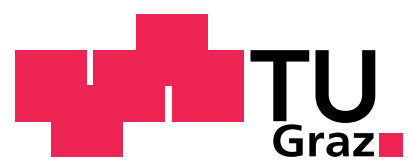

\title{
Regularization of linear inverse problems with total generalized variation
}

\author{
K. Bredies \\ M. Holler
}


SFB sponsors:

- Austrian Science Fund (FWF)

- University of Graz

- Graz University of Technology

- Medical University of Graz

- Government of Styria

- City of Graz

Das Land

Steiermark

Stadt $\mathbf{G} \mathbf{R}$ A Z $\mathbf{Z}$ Wissenschaft 


\title{
Regularization of linear inverse problems with total generalized variation
}

\author{
Kristian Bredies $\quad$ Martin Holler*
}

September 20, 2013

\begin{abstract}
The regularization properties of the total generalized variation (TGV) functional for the solution of linear inverse problems by means of Tikhonov regularization are studied. Considering the associated minimization problem for general symmetric tensor fields, the wellposedness is established in the space of symmetric tensor fields of bounded deformation, a generalization of the space of functions of bounded variation. Convergence for vanishing noise level is shown in a multiple regularization parameter framework in terms of the naturally arising notion of TGV-strict convergence. Finally, some basic properties, in particular non-equivalence for different parameters, are discussed for this notion.
\end{abstract}

Mathematical subject classification (2010): 65L09, 65F22, 65L20, 46G05, 46B26.

Keywords: Linear ill-posed problems, total generalized variation, multiple parameter regularization, symmetric tensor fields, spaces of bounded deformation, a-priori parameter choice.

\section{Introduction}

This paper is concerned with establishing the total generalized variation (TGV) as a regularization functional for ill-posed linear inverse problems

$$
K u=f
$$

in an Tikhonov regularization framework, i.e., the study of the minimization problem

$$
\min _{u \in L^{p}(\Omega)} \frac{1}{q}\left\|K u-f^{\delta}\right\|_{Y}^{q}+\operatorname{TGV}_{\alpha}^{k}(u)
$$

\footnotetext{
*Institute for Mathematics and Scientific Computing, University of Graz, Heinrichstraße 36, A-8010 Graz, Austria. Email: kristian.bredies@uni-graz.at, martin.holler@uni-graz.at.
} 
on a bounded domain $\Omega \subset \mathbb{R}^{d}$ and for noisy data $f^{\delta}$ as well as a suitable $K$. The total generalized variation, introduced in [8], is a functional which is able to regularize, according to the set of parameters $\alpha$, on different scales of smoothness up to a certain order $k$ in terms of the Radon norm. Like its basis, the total variation (TV), it allows for discontinuities but also accounts for higher-order information if $k$ is greater than 1 . The total generalized variation is therefore better suited for modelling piecewise smooth functions than TV which is commonly regarded as a model for piecewise constant functions.

While TGV was first applied as a regularizer in variational imaging for solving denoising problems in $L^{p}(\Omega)$ as well as in several other imaging contexts $[6,7,14,16,17,18]$, the second-order version $\mathrm{TGV}_{\alpha}^{2}$ has been shown to give well-posed Tikhonov-regularized problems

$$
\min _{u \in L^{p}(\Omega)} \frac{1}{2}\left\|K u-f^{\delta}\right\|_{H}^{2}+\mathrm{TGV}_{\alpha}^{2}(u)
$$

where $K$ maps continuously between a Lebesgue space and a Hilbert space [10]. These results heavily rely on the functional-analytic properties of the space of vector fields of bounded deformation $\mathrm{BD}(\Omega)$ which contains all integrable vector fields whose symmetrized derivative is a Radon measure [25, 24]. For establishing generalizations for arbitrary orders $k$ of the TGV-functional, extensions of $\operatorname{BD}(\Omega)$ to symmetric tensor fields of order $l$ have to be considered. However, the functional-analytic properties of these spaces became available only recently [5]. In this paper, the respective results are employed in order to extend the regularization properties to TGV of orders greater than 2. Furthermore, the notion of total generalized variation for scalar functions has also a natural generalization to symmetric tensor fields. While again denoising problems can be solved quite easily and may, for instance, be applied in diffusion tensor imaging (DTI) [27, 26], regularization properties for general tensor order $l$ and arbitrary $k$ are still open. As the treatment of general symmetric tensor fields is inherent for proving well-posedness even in the scalar case, the analysis in the current paper will also cover the tensor case.

The main results can be summarized as follows: First, for any tensor order $l$, differentiation order $k$, any continuous linear operator $K$ mapping from an $L^{p}$-space $(1 \leq p \leq$ $d /(d-1))$ on a bounded Lipschitz domain to a normed space, any $f^{\delta} \in Y$ and $q \geq 1$, a solution of

$$
\min _{u \in L^{p}\left(\Omega, \operatorname{Sym}^{l}\left(\mathbb{R}^{d}\right)\right)} \frac{1}{q}\left\|K u-f^{\delta}\right\|_{Y}^{q}+\operatorname{TGV}_{\alpha}^{k, l}(u)
$$

exists and depends stably on the data $f$. Here, $\mathrm{TGV}_{\alpha}^{k, l}$ denotes the total generalized variation of order $k$ for symmetric tensor fields of order $l$ with weights $\alpha$. For its precise definition, we ask for the reader's patience until Section 3. Second, choosing for each noise level $\delta$ an $f^{\delta}$ such that $\left\|f^{\delta}-f^{\dagger}\right\|_{Y} \leq \delta$ for some exact data $f^{\dagger}$ and letting the parameters $\alpha$ tend to zero in an appropriate manner yields, under respective source conditions, to the convergence towards a $\mathrm{TGV}_{\alpha^{*}}^{k, l}$-minimizing solution with parameters $\alpha^{*}$ for which some can also be infinite. On the way of proving these results, we establish auxiliary properties 
about the structure of TGV which are interesting on its own. The most important are generalizations of [10] to arbitrary orders $k$ and $l$ : We have the probably more convenient minimum representation as a differentiation cascade according to

$$
\operatorname{TGV}_{\alpha}^{k, l}(u)=\min _{\substack{w_{i} \in \operatorname{BD}\left(\Omega, \operatorname{Sym}_{i=1}^{l+i}\left(\mathbb{R}^{d}\right)\right), i=0, \ldots, k, w_{0}=u, w_{k}=0}} \sum_{i=1}^{k} \alpha_{k-i}\left\|\mathcal{E} w_{i-1}-w_{i}\right\|_{\mathcal{M}}
$$

where $\operatorname{BD}\left(\Omega, \operatorname{Sym}^{l}\left(\mathbb{R}^{d}\right)\right)$ is the space of symmetric tensor fields of bounded deformation, $\mathcal{E}$ the weak symmetrized derivative and $\|\cdot\|_{\mathcal{M}}$ denotes the Radon norm. Furthermore, the norm equivalence

$$
\|u\|_{\mathrm{BGV}}=\|u\|_{1}+\mathrm{TGV}_{\alpha}^{k, l}(u) \sim\|u\|_{\mathrm{BD}}=\|u\|_{1}+\|\mathcal{E} u\|_{\mathcal{M}}
$$

is established from which the coincidence of TGV-based Banach spaces with $\operatorname{BD}\left(\Omega, \operatorname{Sym}^{l}\left(\mathbb{R}^{d}\right)\right)$ follows. This, in turn, can be used to obtain $L^{p}$-coercivitiy estimates which are necessary for existence and stability of solutions for the Tikhonov-functional minimization problem (1.1).

It is worth mentioning that, as the result of the great interest in the recent years, the study of regularization functionals in general, non-reflexive Banach spaces is now a wellestablished part of the theory of inverse problems (see, for instance, $[13,21,23]$ and the references therein). In particular, regularization properties of the total variation and total deformation are already known for some time $[1,22]$. While in most of the literature, a single regularization parameter is considered, there have also been some efforts to understand regularization and convergence behaviour for multiple parameters and functionals. However, all efforts aim towards regularization where multiple functionals are summed up according to the regularization parameters $[19,15,12]$ or regularization is achieved by the infimal convolution of two functionals weighted by the parameters [20]. To the best knowledge of the authors, convergence for multiple parameters in a minimization cascade like (1.2) has not been considered so far.

The paper is organized as follows. Section 2 introduces into the concepts which are the basis for the total generalized variation: Symmetric tensor fields and associated spaces. In particular, the needed results about spaces of symmetric tensor fields of bounded deformation are collected. In Section 3, the functional-analytic framework for TGV-regularization is established. In particular the above-mentioned minimum characterization, topological equivalence and coercivity are obtained. Section 4 then deals with showing that TGV can indeed be used as a regularizer for linear inverse problems by first obtaining existence and stability of regularized solutions according to the Tikhonov-functional (1.1) and noisy data and then showing convergence of these solutions to a TGV-minimizing solution as the noise vanishes. As in this case, also the values of the TGV-functional converge, we can speak of TGV-strict convergence. Section 5 discusses this convergence notion which is stronger than weak*-convergence but weaker than strong convergence. Finally, some conclusions are drawn in Section 6. 


\section{Preliminaries}

In the following, we will recall some notation and basic facts about the total generalized variation, mainly for the reader's convenience. More details can be found in $[8,5]$.

\subsection{Basic tensor calculus}

First, let us fix a space dimension $d$ and introduce the tensor framework we are dealing with. As we do not consider tensors in an differential geometric setting, we only consider contravariant tensors, i.e., define the space $\mathcal{T}^{k}\left(\mathbb{R}^{d}\right)$ of tensors of order $k$ as all $k$-linear mappings, i.e.,

$$
\mathcal{T}^{k}\left(\mathbb{R}^{d}\right)=\{\xi: \underbrace{\mathbb{R}^{d} \times \cdots \times \mathbb{R}^{d}}_{k \text { times }} \rightarrow \mathbb{R} \mid \xi k \text {-linear }\} .
$$

Beside the basic vector space operations, we consider the tensor product $\otimes: \mathcal{T}^{k}\left(\mathbb{R}^{d}\right) \times$ $\mathcal{T}^{l}\left(\mathbb{R}^{d}\right) \rightarrow \mathcal{T}^{k+l}\left(\mathbb{R}^{d}\right)$ defined by

$$
(\xi \otimes \eta)\left(a_{1}, \ldots, a_{k+l}\right)=\xi\left(a_{1}, \ldots, a_{k}\right) \eta\left(a_{k+1}, \ldots, a_{k+l}\right)
$$

the reverse ordering ${ }^{-}: \mathcal{T}^{k}\left(\mathbb{R}^{d}\right) \rightarrow \mathcal{T}^{k}\left(\mathbb{R}^{d}\right)$,

$$
\bar{\xi}\left(a_{1}, \ldots, a_{k}\right)=\xi\left(a_{k}, \ldots, a_{1}\right)
$$

as well as, for $k \geq 2$, the trace $\operatorname{tr}: \mathcal{T}^{k}\left(\mathbb{R}^{d}\right) \rightarrow \mathcal{T}^{k-2}\left(\mathbb{R}^{d}\right)$,

$$
\operatorname{tr}(\xi)\left(a_{1}, \ldots, a_{k-2}\right)=\sum_{i=1}^{d} \xi\left(e_{i}, a_{1}, \ldots, a_{k-2}, e_{i}\right) .
$$

A scalar product and Frobenius-type norm for $\mathcal{T}^{k}\left(\mathbb{R}^{d}\right)$ is then given by

$$
\xi \cdot \eta=\operatorname{tr}^{k}(\xi \otimes \bar{\eta}), \quad|\xi|=\sqrt{\xi \cdot \xi},
$$

respectively. Finally, a tensor is symmetric if it is invariant under permutations of the arguments, i.e.,

$$
\xi\left(a_{\pi(1)}, \ldots, a_{\pi(k)}\right)=\xi\left(a_{1}, \ldots, a_{k}\right) \quad \text { for all } a_{1}, \ldots, a_{k} \in \mathbb{R}^{d}, \pi \in S^{k} .
$$

We denote by $\operatorname{Sym}^{k}\left(\mathbb{R}^{d}\right)$ the subspace of symmetric tensors. The symmetrization || $\mid$ : $\mathcal{T}^{k}\left(\mathbb{R}^{d}\right) \rightarrow \operatorname{Sym}^{k}\left(\mathbb{R}^{d}\right)$ given by

$$
(\| \mid \xi)\left(a_{1}, \ldots, a_{k}\right)=\frac{1}{k !} \sum_{\pi \in S^{k}} \xi\left(a_{\pi(1)}, \ldots, a_{\pi(k)}\right)
$$

is then the orthogonal projection onto $\operatorname{Sym}^{k}\left(\mathbb{R}^{d}\right)$. 
For a domain $\Omega \subset \mathbb{R}^{d}$, we call mappings from $\Omega$ to $\mathcal{T}^{k}\left(\mathbb{R}^{d}\right)$ and $\operatorname{Sym}^{k}\left(\mathbb{R}^{d}\right)$ tensor fields and symmetric tensor fields, respectively. The Banach spaces of continuously differentiable (symmetric) tensor fields are then given in the usual way. In the following, we use the Banach space

$$
\begin{aligned}
\mathcal{C}^{m}(\bar{\Omega}, X) & =\{u: \Omega \rightarrow X \mid u \text { is } m \text { times uniformly continuously differentiable }\} \\
\|u\|_{\infty, m} & =\max _{l=0, \ldots, m} \sup _{x \in \Omega}\left|\nabla^{l} \otimes u(x)\right|
\end{aligned}
$$

as well as

$$
\begin{aligned}
& \mathcal{C}_{\mathrm{c}}^{m}(\Omega, X)=\left\{u \in \mathcal{C}^{m}(\bar{\Omega}, X) \mid \operatorname{supp} u \subset \subset \Omega\right\}, \\
& \mathcal{C}_{0}^{m}(\Omega, X)=\overline{\mathcal{C}_{\mathrm{c}}^{m}(\Omega, X)} \text { in } \mathcal{C}^{m}(\bar{\Omega}, X)
\end{aligned}
$$

where $X$ is either $\mathcal{T}^{k}\left(\mathbb{R}^{d}\right)$ or $\operatorname{Sym}^{k}\left(\mathbb{R}^{d}\right)$ and $\nabla^{l} \otimes u$ is the identification of the $l$-th derivative of $u$ with a tensor field $\left(\nabla^{l} \otimes u\right): \Omega \rightarrow \mathcal{T}^{k+l}\left(\mathbb{R}^{d}\right)$ via

$$
\left(\nabla^{l} \otimes u\right)(x)\left(a_{1}, \ldots, a_{k+l}\right)=\mathrm{D} u(x)\left(a_{1}, \ldots, a_{l}\right)\left(a_{l+1}, \ldots, a_{k+1}\right) .
$$

Setting formally $m=\infty$ then means the intersection of the spaces for all $m \geq 0$. Likewise, for a measure $\mu$ on $\Omega$, measurable functions $u: \Omega \rightarrow X$ as well as the corresponding Lebesgue spaces $L_{\mu}^{p}(\Omega, X)$ are also defined the usual way and we drop the subscript if $\mu$ is the $d$-dimensional Lebesgue measure.

Now, turning again to differentiation, note that the divergence $\operatorname{div} u: \Omega \rightarrow \mathcal{T}^{k-1}\left(\mathbb{R}^{d}\right)$ of a differentiable $k$-tensor field $u: \Omega \rightarrow \mathcal{T}^{k}\left(\mathbb{R}^{d}\right)$ is given by

$$
(\operatorname{div} u)=\operatorname{tr}(\nabla \otimes u)
$$

Observe that $\operatorname{div} u$ is symmetric if $u$ is symmetric. Of course, this does not hold for the derivative and we are in particular interested in the symmetrization of the derivative $\mathcal{E} u=\|(\nabla \otimes u)$. For distributions $u \in \mathcal{D}\left(\Omega, \mathcal{T}^{k}\left(\mathbb{R}^{d}\right)\right), \nabla \otimes u \in \mathcal{D}\left(\Omega, \mathcal{T}^{k+1}\left(\mathbb{R}^{d}\right)\right)$ and $\mathcal{E} u \in \mathcal{D}\left(\Omega, \operatorname{Sym}^{k+1}\left(\mathbb{R}^{d}\right)\right)$ are defined in the weak sense by

$$
\begin{aligned}
\langle\nabla \otimes u, \varphi\rangle & =-\langle u, \operatorname{div} \varphi\rangle, & & \varphi \in \mathcal{C}_{\mathrm{c}}^{\infty}\left(\Omega, \mathcal{T}^{k+1}\left(\mathbb{R}^{d}\right)\right), \\
\langle\mathcal{E} u, \varphi\rangle & =-\langle u, \operatorname{div} \varphi\rangle, & & \varphi \in \mathcal{C}_{\mathrm{c}}^{\infty}\left(\Omega, \operatorname{Sym}^{k+1}\left(\mathbb{R}^{d}\right)\right) .
\end{aligned}
$$

This allows to define Sobolev spaces of weakly (symmetrically) differentiable functions. For the analysis of the total generalized variation, we are in particular interested in symmetric tensor fields whose weak symmetrized derivative is a Radon measure which will be described in the following. But first, we recall that by Riesz' representation theorem, the space of Radon measures can be identified with the dual space of continuous functions vanishing at the boundary. We take this as a definition, i.e.,

$$
\mathcal{M}(\Omega, X)=\mathcal{C}_{0}(\Omega, X)^{*}, \quad X \in\left\{\mathcal{T}^{k}\left(\mathbb{R}^{d}\right), \operatorname{Sym}^{k}\left(\mathbb{R}^{d}\right)\right\}
$$


The Radon norm then corresponds to

$$
\|u\|_{\mathcal{M}}=\sup \left\{\langle u, \varphi\rangle \mid \varphi \in \mathcal{C}_{0}(\Omega, X),\|\varphi\|_{\infty} \leq 1\right\}
$$

Besides the convergence associated with the norm, we naturally have a notion of weak*convergence. Also note that then, a distribution $u \in \mathcal{D}(\Omega, X)$ then belongs to $\mathcal{M}(\Omega, X)$ if and only if the supremum of $\langle u, \varphi\rangle$ tested against all $\mathcal{C}_{\mathrm{c}}^{\infty}(\Omega, X)$ with $\|\varphi\|_{\infty} \leq 1$ is finite, as this characterizes whether $u$ can be extended to $\mathcal{C}_{0}(\Omega, X)$ by density.

\subsection{Symmetric tensor fields of bounded deformation}

Definition 2.1. Let $l \in \mathbb{N}, l \geq 0$. The space $\operatorname{BD}\left(\Omega, \operatorname{Sym}^{l}\left(\mathbb{R}^{d}\right)\right)$ of symmetric tensor fields of bounded deformation is defined as the Banach space

$$
\begin{gathered}
\operatorname{BD}\left(\Omega, \operatorname{Sym}^{l}\left(\mathbb{R}^{d}\right)\right)=\left\{u \in L^{1}\left(\Omega, \operatorname{Sym}^{l}\left(\mathbb{R}^{d}\right)\right) \mid \mathcal{E} u \in \mathcal{M}\left(\Omega, \operatorname{Sym}^{l+1}\left(\mathbb{R}^{d}\right)\right)\right\} \\
\|u\|_{\mathrm{BD}}=\|u\|_{1}+\|\mathcal{E} u\|_{\mathcal{M}}
\end{gathered}
$$

Note that $\operatorname{BD}\left(\Omega, \operatorname{Sym}^{l}\left(\mathbb{R}^{d}\right)\right)$ is indeed complete. The Radon norm of $\mathcal{E} u$ can also be expressed by the total deformation

$$
\|\mathcal{E} u\|_{\mathcal{M}}=\operatorname{TD}^{l}(u)=\sup \left\{\int_{\Omega} u \cdot \operatorname{div} \varphi \mathrm{d} x \mid \varphi \in \mathcal{C}_{\mathrm{c}}^{1}\left(\Omega, \operatorname{Sym}^{l+1}\left(\mathbb{R}^{d}\right)\right),\|\varphi\|_{\infty} \leq 1\right\} .
$$

For $l \in\{0,1\}$, these spaces correspond to well-known spaces in the literature:

$$
\mathrm{BD}\left(\Omega, \operatorname{Sym}^{0}\left(\mathbb{R}^{d}\right)\right)=\mathrm{BV}(\Omega), \quad \operatorname{BD}\left(\Omega, \operatorname{Sym}^{1}\left(\mathbb{R}^{d}\right)\right)=\mathrm{BD}(\Omega)
$$

where $\operatorname{BV}(\Omega)$ denotes the space of functions of bounded variation $[2]$ and $\operatorname{BD}(\Omega)$ the space of vector fields of bounded deformation [25], which is studied, for instance, in mathematical plasticity [24]. Also, for scalar functions $u$, we have $\operatorname{TD}^{0}(u)=\operatorname{TV}(u)$. The general case $l \geq 0$ has only recently been introduced in [5]. All spaces of bounded deformation are weaker than an analogous space of bounded variation which considers the full weak derivative instead of its symmetrization. In particular, symmetric tensor fields of bounded deformation are allowed to have jump discontinuities on hypersurfaces.

Analogously to $\operatorname{BV}(\Omega)$, one can define a notion of weak* and strict convergence.

Definition 2.2. A sequence $\left\{u^{n}\right\}$ in $\operatorname{BD}\left(\Omega, \operatorname{Sym}^{l}\left(\mathbb{R}^{d}\right)\right)$ is said to converge to an $u \in$ $\operatorname{BD}\left(\Omega, \operatorname{Sym}^{l}\left(\mathbb{R}^{d}\right)\right)$ in the weak* sense, denoted $u^{n} \stackrel{*}{\rightarrow} u$, if

$$
\lim _{n \rightarrow \infty}\left\|u^{n}-u\right\|_{1}=0 \quad \text { and } \quad \mathcal{E} u^{n} \stackrel{*}{*} \mathcal{E} u \quad \text { in } \quad \mathcal{M}\left(\Omega, \operatorname{Sym}^{l+1}\left(\mathbb{R}^{d}\right)\right) .
$$

It converges $\mathrm{TD}^{l}$-strictly to $u$ if

$$
\lim _{n \rightarrow \infty}\left\|u^{n}-u\right\|_{1}=0 \quad \text { and } \quad \lim _{n \rightarrow \infty} \operatorname{TD}^{l}\left(u^{n}\right)=\operatorname{TD}^{l}(u) .
$$


These notions are motivated by the following embedding and compactness results.

Theorem 2.3. Let $\Omega$ be a bounded Lipschitz domain. Then,

1. $\operatorname{BD}\left(\Omega, \operatorname{Sym}^{k}\left(\mathbb{R}^{d}\right)\right) \hookrightarrow L^{d /(d-1)}\left(\Omega, \operatorname{Sym}^{k}\left(\mathbb{R}^{d}\right)\right)$ continuously and

2. $\operatorname{BD}\left(\Omega, \operatorname{Sym}^{k}\left(\mathbb{R}^{d}\right)\right) \hookrightarrow L^{p}\left(\Omega, \operatorname{Sym}^{k}\left(\mathbb{R}^{d}\right)\right)$ compactly for $1 \leq p<d /(d-1)$.

It is then clear that bounded sequences in $\operatorname{BD}\left(\Omega, \operatorname{Sym}^{k}\left(\mathbb{R}^{d}\right)\right)$ admit a subsequence which converges in the weak*-sense. Also, we see that strict convergence implies weak*convergence. In particular, the embeddings turn weak*-convergent sequences in $\operatorname{BD}\left(\Omega, \operatorname{Sym}^{k}\left(\mathbb{R}^{d}\right)\right)$ to weakly converging sequences in $L^{d /(d-1)}\left(\Omega, \operatorname{Sym}^{k}\left(\mathbb{R}^{d}\right)\right.$ ) (weakly* for $d=1$ ) as well as to strongly converging sequences in $L^{p}\left(\Omega, \operatorname{Sym}^{k}\left(\mathbb{R}^{d}\right)\right)$ for any $p \in[1, d /(d-1)$ [ in a continuous manner.

One can show that $\mathcal{C}^{\infty}\left(\bar{\Omega}, \operatorname{Sym}^{k}\left(\mathbb{R}^{d}\right)\right)$ is dense in $\operatorname{BD}\left(\Omega, \operatorname{Sym}^{k}\left(\mathbb{R}^{d}\right)\right)$ with respect to strict convergence. Like functions of bounded variation, $\operatorname{BD}\left(\Omega, \operatorname{Sym}^{k}\left(\mathbb{R}^{d}\right)\right)$ possess appropriate boundary traces and a Gauss-Green formula is valid.

Theorem 2.4. Let $\Omega \subset \mathbb{R}^{d}$ be a bounded Lipschitz domain. Then, there exists a boundary trace mapping i.e.,

$\gamma \in \mathcal{L}\left(\operatorname{BD}\left(\Omega, \operatorname{Sym}^{l}\left(\mathbb{R}^{d}\right)\right), L^{1}\left(\partial \Omega, \operatorname{Sym}^{l}\left(\mathbb{R}^{d}\right)\right)\right), \quad \gamma u=\left.u\right|_{\partial \Omega} \quad$ for all $\quad u \in \mathcal{C}^{1}\left(\bar{\Omega}, \operatorname{Sym}^{l}\left(\mathbb{R}^{d}\right)\right)$, which is sequentially continuous with respect to strict convergence in $\operatorname{BD}\left(\Omega, \operatorname{Sym}^{l}\left(\mathbb{R}^{d}\right)\right)$ and strong convergence in $L^{1}\left(\partial \Omega, \operatorname{Sym}^{k}\left(\mathbb{R}^{d}\right)\right)$.

For each $u \in \operatorname{BD}\left(\Omega, \operatorname{Sym}^{l}\left(\mathbb{R}^{d}\right)\right)$ and $v \in \mathcal{C}^{1}\left(\bar{\Omega}, \operatorname{Sym}^{l+1}\left(\mathbb{R}^{d}\right)\right)$ the Gauss-Green formula is valid, i.e.

$$
\int_{\Omega} v \mathrm{~d} \mathcal{E} u+\int_{\Omega} u \cdot \operatorname{div} v \mathrm{~d} x=\int_{\partial \Omega}((\gamma u) \otimes \nu) \cdot v \mathrm{~d} \mathcal{H}^{d-1}
$$

where $\nu$ denotes the outward unit normal on $\partial \Omega$.

Moreover, we have a Sobolev-Korn-type inequality which relates the $d /(d-1)$-norm with the Radon-norm of the symmetrized derivative.

Theorem 2.5. Let $\Omega \subset \mathbb{R}^{d}$ be a bounded Lipschitz domain. Then:

1. The kernel of $\mathcal{E}$ in $\mathcal{D}\left(\Omega, \operatorname{Sym}^{l}\left(\mathbb{R}^{d}\right)\right)$ is a finite-dimensional subspace of $\operatorname{Sym}^{l}\left(\mathbb{R}^{d}\right)$-valued polynomials of degree less than $l$.

2. For any continuous projection $R$ in $L^{d /(d-1)}\left(\Omega, \operatorname{Sym}^{l}\left(\mathbb{R}^{d}\right)\right)$ onto $\operatorname{ker}(\mathcal{E})$, there exists a $C>0$ only depending on $\Omega$ and $R$, such that

$$
\|u-R u\|_{d /(d-1)} \leq C\|\mathcal{E} u\|_{\mathcal{M}} \quad \text { for all } \quad u \in \operatorname{BD}\left(\Omega, \operatorname{Sym}^{l}\left(\mathbb{R}^{d}\right)\right) .
$$

Proofs of Theorems 2.3-2.5. See [5]. 
We will also need the result that a distribution is $\operatorname{in} \operatorname{BD}\left(\Omega, \operatorname{Sym}^{l}\left(\mathbb{R}^{d}\right)\right)$ as soon as its symmetrized derivative is a Radon measure.

Theorem 2.6. Let $\Omega \subset \mathbb{R}^{d}$ a bounded Lipschitz domain and $u \in \mathcal{D}\left(\Omega, \operatorname{Sym}^{l}\left(\mathbb{R}^{d}\right)\right)$ such that $\mathcal{E} u \in \mathcal{M}\left(\Omega, \operatorname{Sym}^{l+1}\left(\mathbb{R}^{d}\right)\right)$ in the distributional sense. Then, $u \in \operatorname{BD}\left(\Omega, \operatorname{Sym}^{l}\left(\mathbb{R}^{d}\right)\right)$.

Proof. The proof is shown in the appendix.

Finally, we like to mention higher-order spaces which base on the iterated symmetrized gradient $\mathcal{E}^{k}=\mathcal{E} \circ \ldots \circ \mathcal{E}$.

Definition 2.7. For $k, l \in \mathbb{N}$ with $k \geq 1, l \geq 0$ define the spaces

$$
\begin{gathered}
\operatorname{BD}^{k}\left(\Omega, \operatorname{Sym}^{l}\left(\mathbb{R}^{d}\right)\right)=\left\{u \in L^{1}\left(\Omega, \operatorname{Sym}^{l}\left(\mathbb{R}^{d}\right)\right) \mid \mathcal{E}^{k} u \in \mathcal{M}\left(\Omega, \operatorname{Sym}^{l+k}\left(\mathbb{R}^{d}\right)\right)\right\} \\
\|u\|_{\mathrm{BD}^{k}}=\|u\|_{1}+\left\|\mathcal{E}^{k} u\right\|_{\mathcal{M}}
\end{gathered}
$$

By Theorem 2.6 we have that for a $\operatorname{Sym}^{l}\left(\mathbb{R}^{d}\right)$-valued distribution $u$ on $\Omega, \mathcal{E}^{k} u \in$ $\mathcal{M}\left(\Omega, \operatorname{Sym}^{l+k}\left(\mathbb{R}^{d}\right)\right)$ implies $\mathcal{E}^{k-1} u \in \operatorname{BD}\left(\Omega, \operatorname{Sym}^{l+k-1}\left(\mathbb{R}^{d}\right)\right)$, hence

$$
\left\|\mathcal{E}^{k} u\right\|_{\mathcal{M}}=\operatorname{TD}^{l+k-1}\left(\mathcal{E}^{k-1} u\right)
$$

Naturally, this leads to a higher regularity which generalizes and is thus comparable to higher-order total variation. In particular, the spaces are nested in the sense that larger $k$ leads to smaller spaces.

Let us finally note that by considering weak*-convergence of $\left\{\mathcal{E}^{k} u^{n}\right\}$ and convergence $\left\{\mathrm{TD}^{l+k-1}\left(u^{n}\right)\right\}$, respectively, a notion of weak* and $\mathrm{TD}^{l+k-1}$-strict convergence in $\mathrm{BD}^{k}\left(\Omega, \operatorname{Sym}^{l}\left(\mathbb{R}^{d}\right)\right)$ analogous to Definition 2.2 can be established. It is then immediate that strict convergence implies weak*-convergence and that bounded sets are weakly* sequentially compact.

\section{Functional-analytic properties of TGV}

Having collected the necessary notions, we are now able to define and analyze the total generalized variation for symmetric tensor fields in a functional-analytic framework. As already mentioned, we will not only consider the scalar version introduced in [8], but also study a natural generalization to symmetric tensor fields of order $l$, first introduced in [27] and its underlying spaces. As we will see, this generality is in particular useful for the subsequent proofs.

Definition 3.1. Let $\Omega \subset \mathbb{R}^{d}$ a bounded Lipschitz domain, $l \in \mathbb{N}, k \in \mathbb{N}$ with $k \geq 1, l \geq 0$ and $\alpha=\left(\alpha_{0}, \ldots, \alpha_{k-1}\right)$ with $\alpha_{i}>0$ for each $i=0, \ldots, k-1$ a positive weight vector. The 
total generalized variation of order $k$ of the $l$-symmetric tensor field $u \in L_{\text {loc }}^{1}\left(\Omega, \operatorname{Sym}^{l}\left(\mathbb{R}^{d}\right)\right)$ is then given by

$$
\begin{array}{r}
\operatorname{TGV}_{\alpha}^{k, l}(u)=\sup \left\{\int_{\Omega} u \cdot \operatorname{div}^{k} v \mathrm{~d} x \mid v \in \mathcal{C}_{\mathrm{c}}^{k}\left(\Omega, \operatorname{Sym}^{k+l}\left(\mathbb{R}^{d}\right)\right),\right. \\
\left.\left\|\operatorname{div}^{i} v\right\|_{\infty} \leq \alpha_{i}, i=0, \ldots, k-1\right\} .
\end{array}
$$

The normed space of functions of bounded generalized variation $\operatorname{BGV}^{k}\left(\Omega, \operatorname{Sym}^{l}\left(\mathbb{R}^{d}\right)\right)$ is defined by

$$
\begin{aligned}
\operatorname{BGV}^{k}\left(\Omega, \operatorname{Sym}^{l}\left(\mathbb{R}^{d}\right)\right) & =\left\{u \in L^{1}\left(\Omega, \operatorname{Sym}^{l}\left(\mathbb{R}^{d}\right)\right) \mid \operatorname{TGV}_{\alpha}^{k, l}(u)<\infty\right\}, \\
\|u\|_{\mathrm{BGV}} & =\|u\|_{1}+\operatorname{TGV}_{\alpha}^{k, l}(u) .
\end{aligned}
$$

\section{Remark 3.2.}

1. It is immediate that fixing $k$ and $l$ but changing the weights $\alpha$ in $\operatorname{TGV}_{\alpha}^{k, l}$ yields equivalent norms and hence the same spaces. We therefore denote $\operatorname{BGV}^{k}\left(\Omega, \operatorname{Sym}^{l}\left(\mathbb{R}^{d}\right)\right)$ without $\alpha$.

2. Also note that for $k=1, \operatorname{TGV}_{\alpha}^{k, l}(u)=\alpha_{0} \operatorname{TD}^{l}(u)$ and hence $\operatorname{BGV}^{1}\left(\Omega, \operatorname{Sym}^{l}\left(\mathbb{R}^{d}\right)\right)=$ $\operatorname{BD}\left(\Omega, \operatorname{Sym}^{l}\left(\mathbb{R}^{d}\right)\right)$.

3. Finally, observe that $\mathrm{TGV}_{\alpha}^{k, l} \leq \alpha_{k-1} \mathrm{TD}^{l}$ : As one can see from the definition (2.1), the functional $\alpha_{k-1} \mathrm{TD}^{l}$ can be obtained by replacing the constraint $\|\varphi\|_{\infty} \leq 1$ by $\|\varphi\|_{\infty} \leq \alpha_{k-1}$ in the respective supremum. Now, for each $v \in \mathcal{C}_{\mathrm{c}}^{k}\left(\Omega, \operatorname{Sym}^{k+l}\left(\mathbb{R}^{d}\right)\right)$ which is tested against in (3.1), $\operatorname{div}^{k-1} v$ satisfies $\left\|\operatorname{div}^{k-1} v\right\|_{\infty} \leq \alpha_{k-1}$, hence the supremum can only increase.

We are going to prove, in the following, that the equivalence $\operatorname{BGV}^{k, l}(\Omega)=\operatorname{BD}\left(\Omega, \operatorname{Sym}^{l}\left(\mathbb{R}^{d}\right)\right)$ in the sense of Banach spaces actually holds true for all $k$. As a side-product, coercivity estimates of the type

$$
\|u-R u\|_{d /(d-1)} \leq C \operatorname{TGV}_{\alpha}^{k, l}(u)
$$

where $R$ is a projection in $L^{d /(d-1)}\left(\Omega, \operatorname{Sym}^{l}\left(\mathbb{R}^{d}\right)\right)$ onto $\operatorname{ker}\left(\mathcal{E}^{k}\right)$, will follow.

Throughout this section, we assume that $\mathrm{TGV}_{\alpha}^{k, l}$ is given according to Definition 3.1. In particular, $\Omega$ is a bounded Lipschitz domain, $k, l$ are appropriate orders and $\alpha$ is a positive weight vector.

\subsection{The minimum representation}

We start with rewriting $\mathrm{TGV}_{\alpha}^{k, l}$ in terms of a minimization problem which can be considered dual to (3.1). In order to derive this dual problem, some basic facts about the spaces $\operatorname{BGV}^{k}\left(\Omega, \operatorname{Sym}^{l}\left(\mathbb{R}^{d}\right)\right)$ are needed. 
Proposition 3.3. It holds that

1. $\mathrm{TGV}_{\alpha}^{k, l}$ is lower semi-continuous in $L^{1}\left(\Omega, \operatorname{Sym}^{l}\left(\mathbb{R}^{d}\right)\right)$,

2. $\operatorname{TGV}_{\alpha}^{k, l}$ is a continuous semi-norm on $\operatorname{BGV}^{k}\left(\Omega, \operatorname{Sym}^{l}\left(\mathbb{R}^{d}\right)\right)$ with finite-dimensional ker$n e l \operatorname{ker}\left(\mathcal{E}^{k}\right)$,

3. the space $\operatorname{BGV}^{k}\left(\Omega, \operatorname{Sym}^{l}\left(\mathbb{R}^{d}\right)\right)$ is a Banach space.

Proof. Note that $u \mapsto \int_{\Omega} u \cdot \operatorname{div}^{k} v \mathrm{~d} x$ is a continuous functional on $L^{1}\left(\Omega, \operatorname{Sym}^{l}\left(\mathbb{R}^{d}\right)\right)$ for each $v \in \mathcal{C}_{\mathrm{c}}^{k}\left(\Omega, \operatorname{Sym}^{k+l}\left(\mathbb{R}^{d}\right)\right)$. The functional $\mathrm{TGV}_{\alpha}^{k, l}$ is just the pointwise supremum of a non-empty subset of these $v$ and hence lower semi-continuous.

For the same reason, $\mathrm{TGV}_{\alpha}^{k, l}$ is convex. The positive homogeneity immediately follows from the definition, hence $\mathrm{TGV}_{\alpha}^{k, l}$ is a semi-norm. As

$$
\left|\operatorname{TGV}_{\alpha}^{k, l}\left(u^{1}\right)-\operatorname{TGV}_{\alpha}^{k, l}\left(u^{2}\right)\right| \leq \operatorname{TGV}_{\alpha}^{k, l}\left(u^{1}-u^{2}\right) \leq\left\|u^{1}-u^{2}\right\|_{\mathrm{BGV}}
$$

for each $u^{1}, u^{2} \in \operatorname{BGV}^{k}\left(\Omega, \operatorname{Sym}^{l}\left(\mathbb{R}^{d}\right)\right)$, it is also continuous. Finally, for each $v \in \mathcal{C}_{\mathrm{c}}^{k}\left(\Omega, \operatorname{Sym}^{k+l}\left(\mathbb{R}^{d}\right)\right)$ one can find a $\lambda>0$ such that $\left\|\operatorname{div}^{i}(\lambda v)\right\|_{\infty} \leq \alpha_{i}$ for $i=0, \ldots, k-1$. Hence, $\operatorname{TGV}_{\alpha}^{k, l}(u)=0$ if and only if

$$
\int_{\Omega} u \cdot \operatorname{div}^{k} v \mathrm{~d} x=0 \quad \text { for each } v \in \mathcal{C}_{\mathrm{c}}^{k}\left(\Omega, \operatorname{Sym}^{k+l}\left(\mathbb{R}^{d}\right)\right)
$$

which is equivalent to $u \in \operatorname{ker}\left(\mathcal{E}^{k}\right)$ in the weak sense. As $\operatorname{ker}(\mathcal{E})$ considered on $\mathcal{D}\left(\Omega, \operatorname{Sym}^{l+i}\left(\mathbb{R}^{d}\right)\right)$ is finite-dimensional for each $i=0, \ldots, k-1$, see Theorem 2.5 , the latter has to be finitedimensional.

Finally, it follows from standard arguments that for a lower semi-continuous semi-norm $F$ on a Banach space $X$, the subspace where $F$ is finite equipped with $\|\cdot\|_{X}+F$ is complete. Applied to $L^{1}\left(\Omega, \operatorname{Sym}^{l}\left(\mathbb{R}^{d}\right)\right)$ and $\operatorname{TGV}_{\alpha}^{k, l}$, this yields the third statement.

As a further preparation for employing duality, we note:

Lemma 3.4. Let $i \geq 1$ and $w_{i-1} \in \mathcal{C}_{0}^{i-1}\left(\Omega, \operatorname{Sym}^{l+i-1}\left(\mathbb{R}^{d}\right)\right)^{*}, w_{i} \in \mathcal{C}_{0}^{i}\left(\Omega, \operatorname{Sym}^{l+i}\left(\mathbb{R}^{d}\right)\right)^{*}$ be distributions of order $i-1$ and $i$, respectively. Then

$$
\left\|\mathcal{E} w_{i-1}-w_{i}\right\|_{\mathcal{M}}=\sup \left\{\left\langle w_{i-1}, \operatorname{div} \varphi\right\rangle+\left\langle w_{i}, \varphi\right\rangle \mid \varphi \in \mathcal{C}_{\mathrm{c}}^{i}\left(\Omega, \operatorname{Sym}^{l+i}\left(\mathbb{R}^{d}\right)\right),\|\varphi\|_{\infty} \leq 1\right\}
$$

with the right-hand side being finite if and only if $\mathcal{E} w_{i-1}-w_{i} \in \mathcal{M}\left(\Omega, \operatorname{Sym}^{l+i}\left(\mathbb{R}^{d}\right)\right)$ in the distributional sense.

Proof. Note that in the distributional sense, $\left\langle w_{i}-\mathcal{E} w_{i-1}, \varphi\right\rangle=\left\langle w_{i-1}\right.$, $\left.\operatorname{div} \varphi\right\rangle+\left\langle w_{i}, \varphi\right\rangle$ for all $\varphi \in \mathcal{C}_{\mathrm{c}}^{\infty}\left(\Omega, \operatorname{Sym}^{l+i}\left(\mathbb{R}^{d}\right)\right)$. Since $\mathcal{C}_{\mathrm{c}}^{\infty}\left(\Omega, \operatorname{Sym}^{l+i}\left(\mathbb{R}^{d}\right)\right)$ is dense in $\mathcal{C}_{0}\left(\Omega, \operatorname{Sym}^{l+i}\left(\mathbb{R}^{d}\right)\right)$, the distribution $w_{i}-\mathcal{E} w_{i-1}$ can be extended to an element in $\mathcal{C}_{0}\left(\Omega, \operatorname{Sym}^{l+i}\left(\mathbb{R}^{d}\right)\right)^{*}=$ $\mathcal{M}\left(\Omega, \operatorname{Sym}^{l+i}\left(\mathbb{R}^{d}\right)\right)$ if and only if the supremum in (3.2) is finite. In case of finiteness, it coincides with the Radon norm by definition. 
This enables us to derive the problem which is dual to the supremum in (3.1). We will refer to this problem as the minimum representation of $\mathrm{TGV}_{\alpha}^{k, l}$.

Theorem 3.5. For $\mathrm{TGV}_{\alpha}^{k, l}$ defined according to Definition 3.1, we have for each $u \in$ $L_{\text {loc }}^{1}\left(\Omega, \operatorname{Sym}^{l}\left(\mathbb{R}^{d}\right)\right)$ :

$$
\operatorname{TGV}_{\alpha}^{k, l}(u)=\min _{\substack{w_{i} \in \mathrm{BD}\left(\Omega, \mathrm{Sym}^{l+i}\left(\mathbb{R}^{d}\right)\right), i=0, \ldots, k, w_{0}=u, w_{k}=0}} \sum_{i=1}^{k} \alpha_{k-i}\left\|\mathcal{E} w_{i-1}-w_{i}\right\|_{\mathcal{M}}
$$

with the minimum being finite if and only if $u \in \mathrm{BD}\left(\Omega, \operatorname{Sym}^{l}\left(\mathbb{R}^{d}\right)\right)$ and attained for some $w_{0}, \ldots, w_{k}$ where $w_{i} \in \operatorname{BD}\left(\Omega, \operatorname{Sym}^{l+i}\left(\mathbb{R}^{d}\right)\right)$ for $i=0, \ldots, k$ and $w_{0}=u$ as well as $w_{k}=0$ in case of $u \in \operatorname{BD}\left(\Omega, \operatorname{Sym}^{l}\left(\mathbb{R}^{d}\right)\right)$.

Proof. First, take $u \in L_{\text {loc }}^{1}\left(\Omega, \operatorname{Sym}^{l}\left(\mathbb{R}^{d}\right)\right)$ such that $\operatorname{TGV}_{\alpha}^{k, l}(u)<\infty$. We will employ Fenchel-Rockafellar duality. For this purpose, introduce the Banach spaces

$$
\begin{aligned}
X & =\mathcal{C}_{0}^{1}\left(\Omega, \operatorname{Sym}^{1+l}\left(\mathbb{R}^{d}\right)\right) \times \ldots \times \mathcal{C}_{0}^{k}\left(\Omega, \operatorname{Sym}^{k+l}\left(\mathbb{R}^{d}\right)\right), \\
Y & =\mathcal{C}_{0}^{1}\left(\Omega, \operatorname{Sym}^{1+l}\left(\mathbb{R}^{d}\right)\right) \times \ldots \times \mathcal{C}_{0}^{k-1}\left(\Omega, \operatorname{Sym}^{k-1+l}\left(\mathbb{R}^{d}\right)\right),
\end{aligned}
$$

the linear operator

$$
\Lambda \in \mathcal{L}(X, Y), \quad \Lambda v=\left(\begin{array}{c}
-v_{1}-\operatorname{div} v_{2} \\
\cdots \\
-v_{k-1}-\operatorname{div} v_{k}
\end{array}\right)
$$

and the proper, convex and lower semi-continuous functionals

$$
\begin{aligned}
F: X \rightarrow]-\infty, \infty], & F(v)=-\left\langle u, \operatorname{div} v_{1}\right\rangle+\sum_{i=1}^{k} I_{\left\{\|\cdot\|_{\infty} \leq \alpha_{k-i}\right\}}\left(v_{i}\right), \\
G: Y \rightarrow]-\infty, \infty], & G(w)=I_{\{(0, \ldots, 0)\}}(w) .
\end{aligned}
$$

With these choices, the identity

$$
\operatorname{TGV}_{\alpha}^{k, l}(u)=\sup _{v \in X}-F(v)-G(\Lambda v)
$$

follows from the definition in (3.2). In order to show the representation of $\operatorname{TGV}_{\alpha}^{k, l}(u)$ as in (3.3) we want to obtain

$$
\operatorname{TGV}_{\alpha}^{k, l}(u)=\min _{w^{*} \in Y^{*}} F^{*}\left(-\Lambda^{*} w^{*}\right)+G^{*}\left(w^{*}\right) .
$$


This follows from [3, Corollary 2.3] provided we can show that

$$
Y=\bigcup_{\lambda \geq 0} \lambda(\operatorname{dom}(G)-\Lambda \operatorname{dom}(F))
$$

For this purpose, let $w \in Y$ and define backwards recursively: $v_{k}=0 \in \mathcal{C}_{0}^{k}\left(\Omega, \operatorname{Sym}^{k+l}\left(\mathbb{R}^{d}\right)\right)$, $v_{i}=w_{i}-\operatorname{div} v_{i+1} \in \mathcal{C}_{0}^{i}\left(\Omega, \operatorname{Sym}^{i+l}\left(\mathbb{R}^{d}\right)\right)$ for $i=k-1, \ldots, 1$. Hence, $v \in X$ and $-\Lambda v=w$. Moreover, choosing $\lambda>0$ large enough, one can achieve that $\left\|\lambda^{-1} v\right\|_{\infty} \leq \alpha_{k-i}$ for all $i=1, \ldots, k$, so $\lambda^{-1} v \in \operatorname{dom}(F)$ and since $0 \in \operatorname{dom}(G)$, we get the representation $w=$ $\lambda\left(0-\Lambda \lambda^{-1} v\right)$. Thus, the identity (3.4) holds and the minimum is obtained in $Y^{*}$. Now $Y^{*}$ can be written as

$$
Y^{*}=\mathcal{C}_{0}^{1}\left(\Omega, \operatorname{Sym}^{1+l}\left(\mathbb{R}^{d}\right)\right)^{*} \times \ldots \times \mathcal{C}_{0}^{k-1}\left(\Omega, \operatorname{Sym}^{k-1+l}\left(\mathbb{R}^{d}\right)\right)^{*},
$$

with elements $w^{*}=\left(w_{1}^{*}, \ldots, w_{k-1}^{*}\right), w_{i}^{*} \in \mathcal{C}_{0}^{i}\left(\Omega, \operatorname{Sym}^{i+l}\left(\mathbb{R}^{d}\right)\right)^{*}, 1 \leq i \leq k-1$. Therefore, with $w_{0}^{*}=u$ and $w_{k}^{*}=0$ we get, as $G^{*}=0$,

$$
\begin{aligned}
F^{*}\left(-\Lambda^{*} w^{*}\right)+G^{*}\left(w^{*}\right)= & \sup _{v \in X}\left(\left\langle-\Lambda^{*} w^{*}, v\right\rangle+\left\langle u, \operatorname{div} v_{1}\right\rangle-\sum_{i=1}^{k} I_{\left\{\|\cdot\|_{\infty} \leq \alpha_{k-i}\right\}}\left(v_{i}\right)\right) \\
= & \sup _{\substack{v \in X,\left\|v_{i}\right\|_{\infty} \leq \alpha_{k-i}, i=1, \ldots, k}}\left(\left\langle u, \operatorname{div} v_{1}\right\rangle+\sum_{i=1}^{k-1}\left\langle w_{i}^{*}, \operatorname{div} v_{i+1}\right\rangle+\left\langle w_{i}^{*}, v_{i}\right\rangle\right) \\
= & \sum_{i=1}^{k} \alpha_{k-i}\left(\sup _{v_{i} \in \mathcal{C}_{0}^{i}\left(\Omega, \operatorname{Sym}^{i+l}\left(\mathbb{R}^{d}\right)\right),}^{\left\|v_{i}\right\|_{\infty} \leq 1},\left\langle w_{i-1}^{*}, \operatorname{div} v_{i}\right\rangle+\left\langle w_{i}^{*}, v_{i}\right\rangle\right) .
\end{aligned}
$$

From Lemma 3.4 we know that each supremum is finite and coincides with $\left\|\mathcal{E} w_{i-1}^{*}-w_{i}^{*}\right\|_{\mathcal{M}}$ if and only if $\mathcal{E} w_{i-1}^{*}-w_{i}^{*} \in \mathcal{M}\left(\Omega, \operatorname{Sym}^{k+i}\left(\mathbb{R}^{d}\right)\right)$ for $i=1, \ldots, k$. Then, as $w_{k}^{*}=0$, according to Theorem 2.6, this already yields $w_{k-1}^{*} \in \operatorname{BD}\left(\Omega, \operatorname{Sym}^{k+l-1}\left(\mathbb{R}^{d}\right)\right)$, in particular $w_{k-1}^{*} \in$ $\mathcal{M}\left(\Omega, \operatorname{Sym}^{k+l-1}\left(\mathbb{R}^{d}\right)\right)$. Proceeding inductively, we see that $w_{i}^{*} \in \operatorname{BD}\left(\Omega, \operatorname{Sym}^{k+i}\left(\mathbb{R}^{d}\right)\right)$ for each $i=0, \ldots, k$. Hence, it suffices to take the minimum in (3.4) over all BD-tensor fields which gives (3.3).

In addition, the minimum in (3.3) is finite if $u \in \operatorname{BD}\left(\Omega, \operatorname{Sym}^{l}\left(\mathbb{R}^{d}\right)\right)$. Conversely, if $\operatorname{TD}^{l}(u)=\infty$, also $\left\|\mathcal{E} w_{0}^{*}-w_{1}^{*}\right\|_{\mathcal{M}}=\infty$ for all $w_{1}^{*} \in \operatorname{BD}\left(\Omega, \operatorname{Sym}^{l+1}\left(\mathbb{R}^{d}\right)\right)$. Hence, the minimum in (3.3) has to be $\infty$.

In particular, by Theorem 3.5 we have a rigorous proof of the minimum representation for the scalar version discussed in [8, Remark 3.8]: 
Remark 3.6. For $l=1$ it holds that

$$
\operatorname{TGV}_{\alpha}^{k}(u)=\min _{\substack{w_{i} \in \operatorname{BD}\left(\Omega, \operatorname{Sym}^{i}\left(\mathbb{R}^{d}\right)\right), i=0, \ldots, k, w_{0}=u, w_{k}=0}} \sum_{l=1}^{k} \alpha_{k-l}\left\|\mathcal{E} w_{l-1}-w_{l}\right\|_{\mathcal{M}} .
$$

Remark 3.7. The minimum representation also allows to define $\mathrm{TGV}_{\alpha}^{k, l}$ recursively:

$$
\left\{\begin{aligned}
\operatorname{TGV}_{\alpha_{0}}^{1, l}(u) & =\alpha_{0}\|\mathcal{E} u\|_{\mathcal{M}} \\
\operatorname{TGV}_{\alpha}^{k+1, l}(u) & =\min _{w \in \operatorname{BD}\left(\Omega, \operatorname{Sym}^{l+1}\left(\mathbb{R}^{d}\right)\right)} \alpha_{k}\|\mathcal{E} u-w\|_{\mathcal{M}}+\operatorname{TGV}_{\tilde{\alpha}}^{k, l+1}(w)
\end{aligned}\right.
$$

where $\tilde{\alpha}=\left(\alpha_{0}, \ldots, \alpha_{k-1}\right)$ if $\alpha=\left(\alpha_{0}, \ldots, \alpha_{k}\right)$.

Remark 3.8. The total generalized variation is monotonic with respect to its weights. Let $\alpha, \beta \in \mathbb{R}_{+}^{k}$ be two sets of weights such that $\alpha_{i} \leq \beta_{i}$ for $i=0, \ldots, k-1$. Then, for each admissible $w_{0}, \ldots, w_{k}$ in (3.3) it follows

$$
\sum_{i=1}^{k} \alpha_{k-i}\left\|\mathcal{E} w_{i-1}-w_{i}\right\|_{\mathcal{M}} \leq \sum_{i=1}^{k} \beta_{k-i}\left\|\mathcal{E} w_{i-1}-w_{i}\right\|_{\mathcal{M}}
$$

hence, $\operatorname{TGV}_{\alpha}^{k, l}(u) \leq \operatorname{TGV}_{\beta}^{k, l}(u)$ by taking the minimum on both sides.

\subsection{Topological equivalence and coercivity}

The next step is to examine the spaces $\operatorname{BGV}^{k}\left(\Omega, \operatorname{Sym}^{l}\left(\mathbb{R}^{d}\right)\right)$. Again, our aim is to prove that these space coincide with $\operatorname{BD}\left(\Omega, \operatorname{Sym}^{l}\left(\mathbb{R}^{d}\right)\right)$ for fixed $l$ and all $k \geq 1$. We will proceed inductively with respect to $k$ and hence vary $k, l$ but still leave $\Omega$ fixed. The induction step requires some intermediate estimates as follows.

Lemma 3.9. For each $k \geq 1$ and $l \geq 0$ there exists a constant $C_{1}>0$, only depending on $\Omega, k$ and $l$ such that for each $v \in \operatorname{BD}\left(\Omega, \operatorname{Sym}^{l}\left(\mathbb{R}^{d}\right)\right)$ and $\bar{w} \in \operatorname{ker}\left(\mathrm{TGV}_{\alpha}^{k, l+1}\right) \subset$ $L^{1}\left(\Omega, \operatorname{Sym}^{l+1}\left(\mathbb{R}^{d}\right)\right)$,

$$
\|\mathcal{E}(v)\|_{\mathcal{M}} \leq C_{1}\left(\|\mathcal{E}(v)-\bar{w}\|_{\mathcal{M}}+\|v\|_{1}\right)
$$

Proof. If this is not true for some $k$ and $l$, then there exist $\left(v_{n}\right)_{n \in \mathbb{N}}$ and $\left(\bar{w}_{n}\right)_{n \in \mathbb{N}}$ with $v_{n} \in \operatorname{BD}\left(\Omega, \operatorname{Sym}^{l}\left(\mathbb{R}^{d}\right)\right)$ and $\bar{w}_{n} \in \operatorname{ker}\left(\operatorname{TGV}_{\alpha}^{k, l+1}\right)$ such that

$$
\left\|\mathcal{E}\left(v_{n}\right)\right\|_{\mathcal{M}}=1 \quad \text { and } \quad \frac{1}{n} \geq\left\|v_{n}\right\|_{1}+\left\|\mathcal{E}\left(v_{n}\right)-\bar{w}_{n}\right\|_{\mathcal{M}} .
$$

This implies that $\left(\bar{w}_{n}\right)_{n \in \mathbb{N}}$ is bounded in terms of $\|\cdot\|_{\mathcal{M}}$ in the finite dimensional space $\operatorname{ker}\left(\mathrm{TGV}_{\alpha}^{k, l+1}\right)=\operatorname{ker}\left(\mathcal{E}^{k}\right)$, see Proposition 3.3. Consequently, there exists a subsequence, again denoted by $\left(\bar{w}_{n}\right)_{n \in \mathbb{N}}$ and $\bar{w} \in \operatorname{ker}\left(\mathrm{TGV}_{\alpha}^{k, l+1}\right)$ such that $\bar{w}_{n} \rightarrow \bar{w}$ with respect to $\|\cdot\|_{L^{1}}$. Hence, $\mathcal{E}\left(v_{n}\right) \rightarrow \bar{w}$. Further we have that $v_{n} \rightarrow 0$ and thus, by closedness of the weak symmetrized gradient, $\mathcal{E}\left(v_{n}\right) \rightarrow 0$, which contradicts to $\|\mathcal{E}(v)\|_{\mathcal{M}}=1$. 
For what follows, we like to choose a family of projection operators onto the kernel of $\mathrm{TGV}_{\alpha}^{k, l}=\operatorname{ker}\left(\mathcal{E}^{k}\right)$ (see Proposition 3.3).

Definition 3.10. For each $k \geq 1$ and $l \geq 0$, let $R_{k, l}: L^{d /(d-1)}\left(\Omega, \operatorname{Sym}^{l}\left(\mathbb{R}^{d}\right)\right) \rightarrow \operatorname{ker}\left(\mathcal{E}^{k}\right)$ be a linear, continuous and onto projection.

As $\operatorname{ker}\left(\mathcal{E}^{k}\right)$ (on $\Omega$ and for symmetric tensor fields of order $l$ ) is finite-dimensional, such a $R_{k, l}$ always exists and is not necessarily unique. With the help of these projection, the desired coercivity estimates can be formulated.

Proposition 3.11. For each $k \geq 1$ and $l \geq 0$, there exists a constant $C>0$ such that

$$
\|\mathcal{E} u\|_{\mathcal{M}} \leq C\left(\|u\|_{1}+\operatorname{TGV}_{\alpha}^{k, l}(u)\right) \quad \text { as well as } \quad\left\|u-R_{k, l} u\right\|_{d /(d-1)} \leq C \operatorname{TGV}_{\alpha}^{k, l}(u)
$$

for all $u \in L^{d /(d-1)}\left(\Omega, \operatorname{Sym}^{l}\left(\mathbb{R}^{d}\right)\right)$.

Proof. We prove the result by induction with respect to $k$. In the case $k=1$ and $l \geq 0$ arbitrary, the first inequality is immediate while the second one is equivalent to the SobolevKorn inequality in $\operatorname{BD}\left(\Omega, \operatorname{Sym}^{l}\left(\mathbb{R}^{d}\right)\right.$ ) (see proposition [5, Corollary 4.20]).

Now assume both inequalities hold for a fixed $k$ and each $l \geq 0$ and perform an induction step with respect to $k$, i.e., we fix $l \in \mathbb{N},\left(\alpha_{0}, \ldots, \alpha_{k}\right)$ with $\alpha_{i}>0, \Omega$ as well as $R_{k+1, l}$. We assume that assertion (3.7) holds for $\left(\alpha_{0}, \ldots, \alpha_{k-1}\right)$ and any $l^{\prime} \in \mathbb{N}$.

We will first show the uniform estimate for $\|\mathcal{E} u\|_{\mathcal{M}}$ for which it suffices to consider $u \in \operatorname{BD}\left(\Omega, \operatorname{Sym}^{l}\left(\mathbb{R}^{d}\right)\right)$, as otherwise, according to Theorem $3.5, \operatorname{TGV}_{\alpha}^{k+1, l}(u)=\infty$. Then, with the projection $R_{k, l+1}$, the help of Lemma 3.9, the continuous embeddings

$$
\mathrm{BD}\left(\Omega, \operatorname{Sym}^{l+1}\left(\mathbb{R}^{d}\right)\right) \hookrightarrow L^{d /(d-1)}\left(\Omega, \operatorname{Sym}^{l+1}\left(\mathbb{R}^{d}\right)\right) \hookrightarrow L^{1}\left(\Omega, \operatorname{Sym}^{l+1}\left(\mathbb{R}^{d}\right)\right)
$$

and the induction hypothesis, we can estimate for arbitrary $w \in \operatorname{BD}\left(\Omega, \operatorname{Sym}^{l+1}\left(\mathbb{R}^{d}\right)\right)$,

$$
\begin{aligned}
\|\mathcal{E} u\|_{\mathcal{M}} & \leq C_{1}\left(\left\|\mathcal{E} u-R_{k, l+1} w\right\|_{\mathcal{M}}+\|u\|_{1}\right) \\
& \leq C_{2}\left(\|\mathcal{E} u-w\|_{\mathcal{M}}+\left\|w-R_{k, l+1} w\right\|_{d /(d-1)}+\|u\|_{1}\right) \\
& \leq C_{3}\left(\|\mathcal{E} u-w\|_{\mathcal{M}}+\operatorname{TGV}_{\alpha}^{k, l+1}(w)+\|u\|_{1}\right) \\
& \leq C_{4}\left(\alpha_{k}\|\mathcal{E} u-w\|_{\mathcal{M}}+\operatorname{TGV}_{\alpha}^{k, l+1}(w)+\|u\|_{1}\right)
\end{aligned}
$$

for $C_{1}, C_{2}, C_{3}, C_{4}>0$ suitable. Taking the minimum over all such $w \in \operatorname{BD}\left(\Omega, \operatorname{Sym}^{l+1}\left(\mathbb{R}^{d}\right)\right.$ then yields

$$
\|\mathcal{E} u\|_{\mathcal{M}} \leq C_{3}\left(\|u\|_{1}+\mathrm{TGV}_{\alpha}^{k+1, l}(u)\right)
$$

by virtue of the recursive minimum respresentation (3.6).

In order to show the coercivity estimate, assume that the inequality does not hold true. Then, there is a sequence $\left(u^{n}\right)_{n \in \mathbb{N}}$ in $L^{d /(d-1)}\left(\Omega, \operatorname{Sym}^{l}\left(\mathbb{R}^{d}\right)\right)$ such that

$$
\left\|u^{n}-R_{k+1, l} u^{n}\right\|_{d /(d-1)}=1 \quad \text { and } \quad \frac{1}{n} \geq \operatorname{TGV}_{\alpha}^{k+1, l}\left(u^{n}\right) .
$$


Note that $\operatorname{ker}\left(\mathrm{TGV}_{\alpha}^{k+1, l}\right)=\operatorname{ker}\left(\mathcal{E}^{k+1}\right)=\operatorname{rg}\left(R_{k+1, l}\right)$, hence $\operatorname{TGV}_{\alpha}^{k+1, l}\left(u^{n}-R_{k+1, l} u^{n}\right)=$ $\operatorname{TGV}_{\alpha}^{k+1, l}\left(u^{n}\right)$ for each $n$. Thus, since we already know the first estimate in (3.7) to hold,

$$
\left\|\mathcal{E}\left(u^{n}-R_{k+1, l} u^{n}\right)\right\|_{\mathcal{M}} \leq C_{4}\left(\operatorname{TGV}_{\alpha}^{k+1, l}\left(u^{n}\right)+\left\|u^{n}-R_{k+1, l} u^{n}\right\|_{1}\right),
$$

implying, by continuous embedding, that $\left(u^{n}-R_{k+1, l} u^{n}\right)_{n \in \mathbb{N}}$ is bounded in $\operatorname{BD}\left(\Omega, \operatorname{Sym}^{l}\left(\mathbb{R}^{d}\right)\right)$. By compact embedding (see Proposition 2.3) we may therefore conclude that $u^{n}-R_{k+1, l} u^{n} \rightarrow$ $u^{*}$ in $L^{1}\left(\Omega, \operatorname{Sym}^{l}\left(\mathbb{R}^{d}\right)\right)$ for some subsequence (not relabeled). Moreover, as $R_{k+1, l}\left(u^{n}-\right.$ $\left.R_{k+1, l} u^{n}\right)=0$ for all $n$, the limit has to satisfy $R_{k+1, l} u^{*}=0$. On the other hand, by lower semi-continuity (see Proposition 3.3)

$$
0 \leq \operatorname{TGV}_{\alpha}^{k+1, l}\left(u^{*}\right) \leq \liminf _{n \rightarrow \infty} \operatorname{TGV}_{\alpha}^{k+1, l}\left(u^{n}\right)=0,
$$

hence $u^{*} \in \operatorname{ker}\left(\mathcal{E}^{k+1}\right)=\operatorname{rg}\left(R_{k+1, l}\right)$. Consequently, $\lim _{n \rightarrow \infty} u^{n}-R_{k+1, l} u^{n}=u^{*}=R_{k+1, l} u^{*}=$ 0 . From (3.8) it follows that also $\mathcal{E}\left(u^{n}-R_{k+1, l} u^{n}\right) \rightarrow 0$ in $\mathcal{M}\left(\Omega, \operatorname{Sym}^{l+1}\left(\mathbb{R}^{d}\right)\right)$, so $u^{n}-$ $R_{k+1, l} u^{n} \rightarrow 0$ in $\operatorname{BD}\left(\Omega, \operatorname{Sym}^{l}\left(\mathbb{R}^{d}\right)\right)$ and by continuous embedding also in $L^{d /(d-1)}\left(\Omega, \operatorname{Sym}^{l}\left(\mathbb{R}^{d}\right)\right)$. However, this contradicts $\left\|u^{n}-R_{k+1, l} u^{n}\right\|_{d /(d-1)}=1$ for all $n$, and thus the claimed coercivity has to hold.

Remark 3.12. Note that from the proof one can see that the constant $C$ only depends on $k, l, \alpha, \Omega$ and $R_{k, l}$. This also applies for the $C$ and $c$ appearing in the following. We will, however, not explicitely mention the dependency.

From Proposition 3.11 and Remark 3.2 we immediately get the following corollary:

Corollary 3.13. For $k \geq 1$ and $l \geq 0$ there exist $C, c>0$ such that for all $u \in \operatorname{BD}\left(\Omega, \operatorname{Sym}^{l}\left(\mathbb{R}^{d}\right)\right)$ we have

$$
c\left(\|u\|_{1}+\mathrm{TGV}_{\alpha}^{k, l}(u)\right) \leq\|u\|_{1}+\mathrm{TD}^{l}(u) \leq C\left(\|u\|_{1}+\mathrm{TGV}_{\alpha}^{k, l}(u)\right) .
$$

In particular, $\operatorname{BGV}^{k}\left(\Omega, \operatorname{Sym}^{l}\left(\mathbb{R}^{d}\right)\right)=\operatorname{BD}\left(\Omega, \operatorname{Sym}^{l}\left(\mathbb{R}^{d}\right)\right)$ in the sense of Banach space isomorphy.

This topological equivalence for TGV now allows us to obtain bounds on the $w_{1}, \ldots, w_{k-1}$ in (3.3) as the following two propositions show.

Proposition 3.14. There exists a constant $C>0$ such that for each $u \in \operatorname{BD}\left(\Omega, \operatorname{Sym}^{l}\left(\mathbb{R}^{d}\right)\right)$ and $w_{i} \in \mathrm{BD}\left(\Omega, S y m^{l+i}\left(\mathbb{R}^{d}\right)\right), i=1, \ldots, k-1$ being the minimizers in the minimium representation (3.3) it holds that

$$
\left\|w_{i}\right\|_{1}+\left\|\mathcal{E} w_{i}\right\|_{\mathcal{M}} \leq C\|\mathcal{E} u\|_{\mathcal{M}} \quad \text { for all } i=1, \ldots, k-1
$$


Proof. We will first show the boundedness of the $w_{i}$ in $L^{1}\left(\Omega, \operatorname{Sym}^{l+i}\left(\mathbb{R}^{d}\right)\right)$ by induction with respect to $i$. Note that

$\alpha_{k-1}\left\|w_{1}\right\|_{1} \leq \alpha_{k-1}\left\|\mathcal{E} u-w_{1}\right\|_{\mathcal{M}}+\alpha_{k-1}\|\mathcal{E} u\|_{\mathcal{M}} \leq \operatorname{TGV}_{\alpha}^{k, l}(u)+\alpha_{k-1}\|\mathcal{E} u\|_{\mathcal{M}} \leq 2 \alpha_{k-1}\|\mathcal{E} u\|_{\mathcal{M}}$

as $\operatorname{TGV}_{\alpha}^{k, l}(u) \leq \alpha_{k-1}\|\mathcal{E} u\|_{\mathcal{M}}$ by plugging in zeros in (3.3). Now, assume that, for a $i=1, \ldots, k-2$, it holds that $\left\|w_{i}\right\|_{1} \leq C\|\mathcal{E} u\|_{\mathcal{M}}$ for all $u \in \operatorname{BD}\left(\Omega, \operatorname{Sym}^{l}\left(\mathbb{R}^{d}\right)\right)$ and $C>0$ independent of $u$. Then, by employing the equivalence (3.9) and the minimum definition (3.3),

$$
\begin{aligned}
\alpha_{k-i-1}\left\|w_{i+1}\right\|_{1} & \leq \alpha_{k-i-1}\left\|\mathcal{E} w_{i}-w_{i+1}\right\|_{\mathcal{M}}+\alpha_{k-i-1}\left\|\mathcal{E} w_{i}\right\|_{\mathcal{M}} \\
& \leq \operatorname{TGV}_{\alpha}^{k, l}(u)+C \operatorname{TGV}_{\left(\alpha_{0}, \ldots, \alpha_{k-i-1}\right)}^{k-i, l+i}\left(w_{i}\right)+C\left\|w_{i}\right\|_{1} \\
& \leq\left(\sum_{j=1}^{k} \alpha_{k-j}\left\|\mathcal{E} w_{j-1}-w_{j}\right\|_{\mathcal{M}}\right)+C\left(\sum_{j=i+1}^{k} \alpha_{k-j}\left\|\mathcal{E} w_{j-1}-w_{j}\right\|_{\mathcal{M}}\right)+C\|\mathcal{E} u\|_{\mathcal{M}} \\
& \leq C \operatorname{TGV}_{\alpha}^{k, l}(u)+C\|\mathcal{E} u\|_{\mathcal{M}} \leq C\|\mathcal{E} u\|_{\mathcal{M}}
\end{aligned}
$$

which implies the induction step. Hence, $\left\|w_{i}\right\|_{1}$ is bounded in terms of $C\|\mathcal{E} u\|_{\mathcal{M}}$ for all $i=1, \ldots, k-1$ and also for $i=k$ as $w_{k}=0$. Finally, for $i=1, \ldots, k-1$,

$$
\begin{aligned}
\alpha_{k-i-1}\left\|\mathcal{E} w_{i}\right\|_{\mathcal{M}} & \leq \alpha_{k-i-1}\left\|\mathcal{E} w_{i}-w_{i+1}\right\|_{\mathcal{M}}+\alpha_{k-i-1}\left\|w_{i+1}\right\|_{1} \\
& \leq \operatorname{TGV}_{\alpha}^{k, l}(u)+\alpha_{k-i-1}\left\|w_{i+1}\right\|_{1} \leq \alpha_{k-1}\|\mathcal{E} u\|_{\mathcal{M}}+C\|\mathcal{E} u\|_{\mathcal{M}} \leq C\|\mathcal{E} u\|_{\mathcal{M}}
\end{aligned}
$$

which implies the desired estimate.

This estimate can be interpreted as stability of the optimal higher-order information $w_{i}$ subtracted from the respective symmetrized gradients $\mathcal{E} w_{i-1}$ in the differentiation cascade. In case the $w_{1}, \ldots, w_{k-1}$ are not optimal, they still can be bounded if the sum in (3.3) is bounded.

Proposition 3.15. Let, for $i=0, \ldots, k-1,\left\{w_{i}^{n}\right\}$ be sequences in $\operatorname{BD}\left(\Omega, \operatorname{Sym}^{l+i}\left(\mathbb{R}^{d}\right)\right)$ and denote by $\left\{w_{k}^{n}\right\}=0$ the zero sequence in $\operatorname{BD}\left(\Omega, \operatorname{Sym}^{k+l}\left(\mathbb{R}^{d}\right)\right)$. If the sequences

$$
\left\{\left\|\mathcal{E} w_{0}^{n}\right\|_{\mathcal{M}}\right\} \quad \text { and } \quad\left\{\sum_{i=1}^{k} \alpha_{k-i}\left\|\mathcal{E} w_{i-1}^{n}-w_{i}^{n}\right\|_{\mathcal{M}}\right\}
$$

are bounded, then $\left\{w_{i}^{n}\right\}$ are bounded in $\operatorname{BD}\left(\Omega, \operatorname{Sym}^{l+i}\left(\mathbb{R}^{d}\right)\right)$ for $i=1, \ldots, k-1$.

Proof. We show that for each $i=1, \ldots, k-1$, it follows from $\left\{\left\|\mathcal{E} w_{i-1}^{n}\right\|_{\mathcal{M}}\right\}$ bounded that $\left\{w_{i}^{n}\right\}$ is bounded in $\operatorname{BD}\left(\Omega, \operatorname{Sym}^{l+i}\left(\mathbb{R}^{d}\right)\right)$. Let $1 \leq i \leq k-1$ and assume that $\left\{\left\|\mathcal{E} w_{i-1}^{n}\right\|_{\mathcal{M}}\right\}$ is bounded. We have, with $\tilde{\alpha}=\left(\alpha_{0}, \ldots, \alpha_{k-i-1}\right)$, that for each $n$,

$$
\alpha_{k-i}\left\|\mathcal{E} w_{i-1}^{n}-w_{i}^{n}\right\|_{\mathcal{M}}+\operatorname{TGV}_{\tilde{\alpha}}^{k-i, l+i}\left(w_{i}^{n}\right) \leq \sum_{i=1}^{k} \alpha_{k-i}\left\|\mathcal{E} w_{i-1}^{n}-w_{i}^{n}\right\|_{\mathcal{M}},
$$


hence $\left\{\left\|\mathcal{E} w_{i-1}^{n}-w_{i}^{n}\right\|_{\mathcal{M}}\right\}$ and $\left\{\operatorname{TGV}_{\tilde{\alpha}}^{k-i, l+i}\left(w_{i}^{n}\right)\right\}$ are bounded sequences. As $\left\{\left\|\mathcal{E} w_{i-1}^{n}\right\|_{\mathcal{M}}\right\}$ is bounded, $\left\{\left\|w_{i}^{n}\right\|_{1}\right\}$ is bounded and by the norm equivalence result (3.9), $\left\{w_{i}^{n}\right\}$ is bounded in $\operatorname{BD}\left(\Omega, \operatorname{Sym}^{l+i}\left(\mathbb{R}^{d}\right)\right)$. Induction then shows the result.

\section{Regularization of linear inverse problems with TGV}

\subsection{Existence and stability}

We are now able to prove existence and stability for TGV-regularized problems. We start with a general existence result and first note a sufficient condition on $F$ for $F+\mathrm{TGV}_{\alpha}^{k, l}$ to be coercive in appropriate $L^{p}$-spaces. Again, we leave $k$ and $l$ as well as $\Omega$ fixed, the latter being a bounded Lipschitz domain.

Proposition 4.1. Let $p \in\left[1, \infty\left[\right.\right.$ with $p \leq d /(d-1)$ and $\left.\left.F: L^{p}\left(\Omega, \operatorname{Sym}^{l}\left(\mathbb{R}^{d}\right)\right) \rightarrow\right]-\infty, \infty\right]$. If

$$
\left\{\begin{array}{l}
\bullet F \text { is bounded from below and } \\
\text { - there exists an onto projection } R: L^{d /(d-1)}\left(\Omega, \operatorname{Sym}^{l}\left(\mathbb{R}^{d}\right)\right) \rightarrow \operatorname{ker}\left(\mathcal{E}^{k}\right) \text { such } \\
\text { that for each sequence }\left\{u^{n}\right\} \text { in } L^{p}\left(\Omega, \operatorname{Sym}^{l}\left(\mathbb{R}^{d}\right)\right) \text { it holds that } \\
\left\|R u^{n}\right\|_{d /(d-1)} \rightarrow \infty \text { and }\left\{\left\|u^{n}-R u^{n}\right\|_{d /(d-1)}\right\} \text { bounded } \Rightarrow F\left(u^{n}\right) \rightarrow \infty,
\end{array}\right.
$$

then $F+\mathrm{TGV}_{\alpha}^{k, l}$ is coercive in $L^{p}\left(\Omega, \operatorname{Sym}^{l}\left(\mathbb{R}^{d}\right)\right)$.

Proof. We show for each sequence $\left\{u^{n}\right\}$ in $L^{p}\left(\Omega, \operatorname{Sym}^{l}\left(\mathbb{R}^{d}\right)\right)$ that if $\left\{\left(F+\operatorname{TGV}_{\alpha}^{k, l}\right)\left(u^{n}\right)\right\}$ is bounded, then $\left\{u^{n}\right\}$ is bounded. Let such a sequence be given. Then, as $F$ is bounded from below, it follows that $\left\{F\left(u^{n}\right)\right\}$ as well as $\left\{\operatorname{TGV}_{\alpha}^{k, l}\left(u^{n}\right)\right\}$ is bounded. The latter implies that each $u^{n} \in L^{d /(d-1)}\left(\Omega, \operatorname{Sym}^{l}\left(\mathbb{R}^{d}\right)\right.$ ) (see Theorem 2.3). For the projection $R$ chosen in condition (4.1) for $F$, there exists a constant $C>0$ such that $\left\|u^{n}-R u^{n}\right\|_{d /(d-1)} \leq$ $C \operatorname{TGV}_{\alpha}^{k, l}\left(u^{n}\right)$ for all $n$ (see Proposition 3.11). Hence, $\left\{\left\|u^{n}-R u^{n}\right\|_{d /(d-1)}\right\}$ is bounded. Moreover $\left\{\left\|R u^{n}\right\|_{d /(d-1)}\right\}$ has to be bounded as otherwise, (4.1) yields that $\left\{F\left(u^{n}\right)\right\}$ is unbounded which is a contradiction. Finally, the continuous embedding of Lebesgue spaces gives that $\left\{u^{n}\right\}$ is bounded in $L^{p}\left(\Omega, \operatorname{Sym}^{l}\left(\mathbb{R}^{d}\right)\right)$.

With the coercivity of $F+\mathrm{TGV}_{\alpha}^{k, l}$, existence of minimizers is immediate. We present a proof for completeness.

Theorem 4.2. Let $p \in[1, \infty[$ with $p \leq d /(d-1)$ and assume that

1. $\left.\left.F: L^{p}\left(\Omega, \operatorname{Sym}^{l}\left(\mathbb{R}^{d}\right)\right) \rightarrow\right]-\infty, \infty\right]$ is proper, convex, lower semi-continuous,

2. $F$ is coercive in the sense of (4.1).

Then, there exists a solution of the problem

$$
\min _{u \in L^{p}\left(\Omega, \operatorname{Sym}^{l}\left(\mathbb{R}^{d}\right)\right)} F(u)+\operatorname{TGV}_{\alpha}^{k, l}(u) .
$$

In case that there is a $u \in \operatorname{BD}\left(\Omega, \operatorname{Sym}^{l}\left(\mathbb{R}^{d}\right)\right)$ such that $F(u)<\infty$, the minimum is finite. 
Proof. Note that $\operatorname{TGV}_{\alpha}^{k, l}(u)$ is finite if and only if $u \in \operatorname{BD}\left(\Omega, \operatorname{Sym}^{l}\left(\mathbb{R}^{d}\right)\right)$. Thus, if $F(u)=$ $\infty$ for all $u \in \operatorname{BD}\left(\Omega, \operatorname{Sym}^{l}\left(\mathbb{R}^{d}\right)\right)$, the functional is constant $\infty$ and a minimizer trivially exists. Hence, assume in the following that $F(u)<\infty$ for some $u \in \operatorname{BD}\left(\Omega, \operatorname{Sym}^{l}\left(\mathbb{R}^{d}\right)\right)$. Now, denote by $G=F+\mathrm{TGV}_{\alpha}^{k, l}$ and consider a minimizing sequence $\left\{u^{n}\right\}$ for $G$ which exists since $G$ is bounded from below.

Now, applying Proposition 4.1 for a $p^{\prime} \in[p, d /(d-1)]$ and $p^{\prime}>1$, it follows that there exists a weakly convergent subsequence of $\left\{u^{n}\right\}$ (not relabeled) with limit $u^{*} \in$ $L^{p}\left(\Omega, \operatorname{Sym}^{l}\left(\mathbb{R}^{d}\right)\right)$. As $G$ is convex and lower semi-continuous (see Proposition 3.9), we deduce that $u^{*}$ is a minimizer by weak lower semi-continuity. As $G$ is proper, the minimum must be finite.

This result can immediately be applied to linear inverse problems.

Corollary 4.3. For each $K: L^{p}\left(\Omega, \operatorname{Sym}^{l}\left(\mathbb{R}^{d}\right)\right) \rightarrow Y$ linear and continuous in some normed space $Y$, each $f \in Y$ and each $q \in[1, \infty[$ there exists a minimizer of the corresponding Tikhonov functional, i.e., a solution of

$$
\min _{u \in L^{p}\left(\Omega, \operatorname{Sym}^{l}\left(\mathbb{R}^{d}\right)\right)} \frac{1}{q}\|K u-f\|_{Y}^{q}+\operatorname{TGV}_{\alpha}^{k, l}(u) .
$$

Proof. Denote by $F(u)=\frac{1}{q}\|K u-f\|_{Y}^{q}$ and $G=F+\mathrm{TGV}_{\alpha}^{k, l}$. Observe that both $X=$ $\operatorname{ker}(K) \cap \operatorname{ker}\left(\mathcal{E}^{k}\right)$ and

$$
X^{\perp}=\left\{u \in L^{p}\left(\Omega, \operatorname{Sym}^{l}\left(\mathbb{R}^{d}\right)\right) \mid \int_{\Omega} u \cdot v \mathrm{~d} x=0 \text { for all } v \in X\right\}
$$

are closed subspaces in $L^{p}\left(\Omega, \operatorname{Sym}^{l}\left(\mathbb{R}^{d}\right)\right)$ : Being a subset of $\operatorname{ker}\left(\mathcal{E}^{k}\right)$ which is a finitedimensional space of polynomials, it is a finite-dimensional subspace of both $L^{p}\left(\Omega, \operatorname{Sym}^{l}\left(\mathbb{R}^{d}\right)\right)$ and its dual space $L^{p^{*}}\left(\Omega, \operatorname{Sym}^{l}\left(\mathbb{R}^{d}\right)\right)$. It is moreover easy to see that $X^{\perp}$ complements $X$ in $L^{p}\left(\Omega, \operatorname{Sym}^{l}\left(\mathbb{R}^{d}\right)\right)$. Since $G(u+v)=G(u)$ for each $u \in L^{p}\left(\Omega, \operatorname{Sym}^{l}\left(\mathbb{R}^{d}\right)\right)$ and each $v \in X$, it is therefore sufficient to minimize $G$ over $X^{\perp}$.

Hence, consider $\tilde{F}=F+I_{X^{\perp}}$ which is proper, convex lower semi-continuous and bounded from below. To verify the coercivity condition $(4.1)$, let $R: L^{d /(d-1)}\left(\Omega, \operatorname{Sym}^{l}\left(\mathbb{R}^{d}\right)\right) \rightarrow$ $\operatorname{ker}\left(\mathcal{E}^{k}\right)$ be an arbitrary continuous and onto projection. Suppose that $\left\{u^{n}\right\}$ is such that $\left\|R u^{n}\right\|_{d /(d-1)} \rightarrow \infty$ and $\left\{\left\|u^{n}-R u^{n}\right\|_{d /(d-1)}\right\}$ is bounded. First, assume that $u^{n} \in X^{\perp}$ for each $n$. Then, as $K$ is injective on the finite-dimensional space $\operatorname{ker}\left(\mathcal{E}^{k}\right) \cap X^{\perp}$, there is a constant $C>0$ independent of $n$ such that $\left\|R u^{n}\right\|_{d /(d-1)} \leq C\left\|K R u^{n}\right\|_{Y}$ for all $n$. Consequently, one can find a $M>0$ such that

$$
C^{-1}\left\|R u^{n}\right\|_{d /(d-1)}-M \leq\left\|K R u^{n}\right\|_{Y}-\|K\|\left\|u^{n}-R u^{n}\right\|_{d /(d-1)}-\|f\|_{Y} \leq\left\|K u^{n}-f\right\|_{Y}
$$


for all $n$. As the left-hand side tends to $\infty$, the right-hand side must also, hence $\tilde{F}\left(u^{n}\right)=$ $F\left(u^{n}\right) \rightarrow \infty$. Now, suppose $u^{n} \notin X^{\perp}$ for some $n$. Note that $u^{n} \notin X^{\perp}$ implies $\tilde{F}\left(u^{n}\right)=\infty$, so we can apply the above argument to the subsequence of $\left\{u^{n}\right\}$ corresponding to the elements in $X^{\perp}$ to achieve that $\tilde{F}\left(u^{n}\right) \rightarrow \infty$ for the whole sequence. The application of Theorem 4.2 then yields the desired statement.

Remark 4.4. In case the norm $\|\cdot\|_{Y}$ is strictly convex, it is immediate that $K$ injective implies that solutions of (4.2) are unique. In the general case, uniqueness may fail since $\mathrm{TGV}_{\alpha}^{k, l}$ is not strictly convex.

We will now turn to stability and regularization properties. The following is a consequence of abstract standard results.

Theorem 4.5. The solutions of (4.2) are stable in the following sense: For a sequence $\left\{f^{n}\right\}$ which converges to some $f$ in $Y$, there is a subsequence $\left\{u^{n_{\nu}}\right\}$ of solutions $\left\{u^{n}\right\}$, where each $u^{n}$ solves (4.2) with data $f^{n}$, such that $\left\{u^{n_{\nu}}\right\}$ weakly* in $\operatorname{BD}\left(\Omega, \operatorname{Sym}^{l}\left(\mathbb{R}^{d}\right)\right)$ to a solution $u^{*}$ of (4.2) with data $f$. Each sequence of bounded solutions $\left\{u^{n}\right\}$ possesses such a subsequence.

Moreover, for a weak ${ }^{*}$ convergent subsequence of solutions $u^{n_{\nu}} \stackrel{*}{\rightarrow} u^{*}$ in $\mathrm{BD}\left(\Omega, \operatorname{Sym}^{l}\left(\mathbb{R}^{d}\right)\right)$ we have that

$$
\mathrm{TGV}_{\alpha}^{k, l}\left(u^{n_{\nu}}\right) \rightarrow \mathrm{TGV}_{\alpha}^{k, l}\left(u^{*}\right) \quad \text { as } \quad \nu \rightarrow \infty .
$$

Proof. The existence of a subsequence with the desired properties is a direct consequence of [13, Theorem 3.2] applied to $\mathcal{R}=\mathrm{TGV}_{\alpha}^{k, l}+I_{X^{\perp}}$ from Corollary 4.3 and weak* convergence in $\operatorname{BD}\left(\Omega, \operatorname{Sym}^{l}\left(\mathbb{R}^{d}\right)\right)$. Moreover, choosing $\mathcal{R}=\mathrm{TGV}_{\alpha}^{k, l}$ and noting that bounded sequences in $\operatorname{BD}\left(\Omega, \operatorname{Sym}^{l}\left(\mathbb{R}^{d}\right)\right)$ admit weakly* converging subsequences, we see from the proof of the same theorem that the limit $u^{*}$ has to be a solution of (4.2) with data $f$. This also holds true for $\operatorname{TGV}_{\alpha}^{k, l}\left(u^{n_{\nu}}\right) \rightarrow \operatorname{TGV}_{\alpha}^{k, l}\left(u^{*}\right)$ whenever $u^{n_{\nu}} \stackrel{*}{\rightarrow} u^{*}$ as $\nu \rightarrow \infty$.

We close the discussion on existence and stability by some immediate observations concerning special cases of the above theorem.

Remark 4.6.

1. If $\operatorname{ker}(K) \cap \operatorname{ker}\left(\mathcal{E}^{k}\right)=\{0\}$, then each sequence of solutions $\left\{u^{n}\right\}$ with bounded data $\left\{f^{n}\right\}$ is bounded. Otherwise, the sequence of solutions constructed in the proof of Corollary 4.3, i.e. where $u^{n} \in X^{\perp}$ for each $n$, is bounded.

2. If $1 \leq p<d /(d-1)$, then weak* convergence can be replaced by strong convergence in $L^{p}\left(\Omega, \operatorname{Sym}^{l}\left(\mathbb{R}^{d}\right)\right)$ as $\operatorname{BD}\left(\Omega, \operatorname{Sym}^{l}\left(\mathbb{R}^{d}\right)\right) \hookrightarrow L^{p}\left(\Omega, \operatorname{Sym}^{l}\left(\mathbb{R}^{d}\right)\right)$ is compact (see Theorem 2.3).

3. If the solution $u^{*}$ of (4.2) for data $f$ is unique, then the whole sequence $\left\{u^{n}\right\}$ converges weakly* to $u^{*}$. 


\subsection{Convergence for vanishing noise level}

Likewise, regularization properties can be noted. These are again based on the results in [13]. However, a slight modification accounting for multiple regularization parameters has to be made.

Let us first take a look at how the total generalized variation behaves for fixed argument and varying parameters. For this purpose, we like also to allow the weights $\infty$ : We will therefore denote by

$$
\alpha\|\cdot\|=I_{\{0\}} \quad \text { if } \quad \alpha=\infty
$$

for any norm on a normed space. Then, for $\left.\left.\alpha=\left(\alpha_{0}, \ldots, \alpha_{k-1}\right) \in\right] 0, \infty\right]^{k}$ we set

$$
\operatorname{TGV}_{\alpha}^{k, l}(u)=\min _{\substack{w_{i} \in \operatorname{BD}\left(\Omega, \operatorname{Sym}_{i+i}^{l+i}\left(\mathbb{R}^{d}\right)\right), i=0, \ldots, k, w_{0}=u, w_{k}=0}} \sum_{i=1}^{k} \alpha_{k-i}\left\|\mathcal{E} w_{i-1}-w_{i}\right\|_{\mathcal{M}}
$$

which is obviously well-defined (by the direct method, using the boundedness result in Proposition 3.15) and coincides with (3.3) for positive weights. Also, we agree to set $\mathrm{TGV}^{0, l}=0$.

Observe that a coefficient $\alpha_{k-i}$ being infinity can be interpreted as a restriction $w_{i}=$ $\mathcal{E} w_{i-1}$ and consequently, a way of introducing powers of $\mathcal{E}$ into (3.3). Indeed, setting $i_{0}=0$, denoting by $i_{1}, \ldots, i_{M}$ the elements of the set $\left\{i \mid 1 \leq i \leq k, \alpha_{k-i}<\infty\right\}$ in a strictly increasing manner as well as letting $i_{M+1}=k,(4.4)$ can be rewritten as

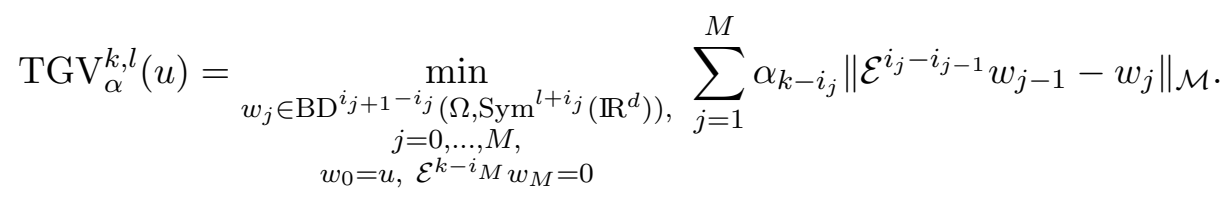

The observation now is that fixing $u$ and choosing a convergent sequence of parameters $\alpha$, the corresponding values of the TGV-functional converges, even if one allows $\infty$ in the limit.

Proposition 4.7. Let $u \in \operatorname{BD}\left(\Omega, \operatorname{Sym}^{l}\left(\mathbb{R}^{d}\right)\right)$ and $\left\{\alpha^{n}\right\}$ be a sequence in $] 0, \infty\left[^{k}\right.$ which converges to some $\left.\left.\alpha^{*} \in\right] 0, \infty\right]^{k}$. Then, $\operatorname{TGV}_{\alpha^{n}}^{k, l}(u) \rightarrow \operatorname{TGV}_{\alpha^{*}}^{k, l}(u)$ as $n \rightarrow \infty$.

Additionally, for each sequence $\left\{u^{n}\right\}$ in $\operatorname{BD}\left(\Omega, \operatorname{Sym}^{l}\left(\mathbb{R}^{d}\right)\right)$ with $u^{n} \stackrel{*}{\rightarrow} u$ it holds that $\operatorname{TGV}_{\alpha^{*}}^{k, l}(u) \leq \liminf \operatorname{in}_{n \rightarrow \infty} \operatorname{TGV}_{\alpha^{n}}^{k, l}\left(u^{n}\right)$.

Proof. We will first bound the limes superior by $\operatorname{TGV}_{\alpha^{*}}^{k, l}(u)$. For this purpose, we may assume that $\operatorname{TGV}_{\alpha^{*}}^{k, l}(u)<\infty$. This implies in particular that there are feasible $w_{0}, \ldots, w_{k}$ such that the minimum in (4.4) is attained. Denoting by $M=\left\{i \mid 1 \leq i \leq k, \alpha_{k-i}^{*}<\infty\right\}$ and introducing $c_{n}=\max _{i \in M} \alpha_{k-i}^{n} / \alpha_{k-i}^{*}$ (where we agree to set $c_{n}=1$ if $M=\emptyset$ ), one gets

$$
\operatorname{TGV}_{\alpha^{n}}^{k, l}(u) \leq \sum_{i=1}^{k} \alpha_{k-i}^{n}\left\|\mathcal{E} w_{i-1}-w_{i}\right\|_{\mathcal{M}} \leq c_{n} \sum_{i=1}^{k} \alpha_{k-i}^{*}\left\|\mathcal{E} w_{i-1}-w_{i}\right\|_{\mathcal{M}}=c_{n} \operatorname{TGV}_{\alpha^{*}}^{k, l}(u)
$$


Due to convergence of $\left\{\alpha^{n}\right\}$, we have $c_{n} \rightarrow 1$ as $n \rightarrow \infty$ and consequently,

$$
\limsup _{n \rightarrow \infty} \operatorname{TGV}_{\alpha^{n}}^{k, l}(u) \leq \operatorname{TGV}_{\alpha^{*}}^{k, l}(u) .
$$

An estimate on the limes inferior will follow from the proof of the addition by taking constant sequences. Hence, let $\left\{u^{n}\right\}$ be a sequence in $\operatorname{BD}\left(\Omega, \operatorname{Sym}^{l}\left(\mathbb{R}^{d}\right)\right)$ which converges in the weak* sense to $u$. We may assume that $\liminf _{n \rightarrow \infty} \operatorname{TGV}_{\alpha^{n}}^{k, l}(u)<\infty$. Introduce $w_{0}^{n}, \ldots, w_{k}^{n}$ the admissible tensor fields which admit the minimum in (3.3) for $u^{n}$ and $\alpha^{n}$, respectively, as well as $\underline{\alpha}$ the weights according to $\underline{\alpha}_{i}=\inf _{n \in \mathbb{N}} \alpha_{i}^{n}$ (which are positive due to $\alpha_{i}^{n} \rightarrow \alpha_{i}^{*}$ as $n \rightarrow \infty$ and $\alpha_{i}^{*}$ being positive). Hence,

$$
\begin{aligned}
\liminf _{n \rightarrow \infty} \sum_{i=1}^{k} \underline{\alpha}_{k-i}\left\|\mathcal{E} w_{i-1}^{n}-w_{i}^{n}\right\|_{\mathcal{M}} \leq \liminf _{n \rightarrow \infty} \sum_{i=1}^{k} \alpha_{k-i}^{n} \| \mathcal{E} w_{i-1}^{n}- & w_{i}^{n} \|_{\mathcal{M}} \\
& =\liminf _{n \rightarrow \infty} \operatorname{TGV}_{\alpha_{n}}^{k, l}\left(u^{n}\right)<\infty .
\end{aligned}
$$

It follows that the left-hand side is bounded for infinitely many $n$ and as $\mathcal{E} w_{0}^{n}=\mathcal{E} u^{n}$ which is bounded, so Proposition 3.15 implies the existence of a subsequence of $\left\{\left(w_{0}^{n}, \ldots, w_{k}^{n}\right)\right\}$ (not relabeled) such that each $\left\{w_{i}^{n}\right\}$ is bounded in $\operatorname{BD}\left(\Omega, \operatorname{Sym}^{l+i}\left(\mathbb{R}^{d}\right)\right)$ and by weak* sequential compactness of bounded sets, one can also assume that $w_{i}^{n} \stackrel{*}{\rightarrow} w_{i}^{*}$ for some $w_{i}^{*} \in \operatorname{BD}\left(\Omega, \operatorname{Sym}^{l+i}\left(\mathbb{R}^{d}\right)\right)$ and each $i=0, \ldots, k$. Now, for an $i$ with $\alpha_{k-i}^{*}<\infty$ we get from weak* $^{*}$ lower semicontinuity and the invariance of the limes inferior under multiplication with a positive, convergent sequence that

$$
\alpha_{k-i}^{*}\left\|\mathcal{E} w_{i-1}^{*}-w_{i}^{*}\right\|_{\mathcal{M}} \leq \liminf _{n \rightarrow \infty} \alpha_{k-i}^{n}\left\|\mathcal{E} w_{i-1}^{n}-w_{i}^{n}\right\|_{\mathcal{M}}
$$

For $i$ such that $\alpha_{k-i}^{*}=\infty$ we have that $\left\{\alpha_{k-i}^{n}\left\|\mathcal{E} w_{i-1}^{n}-w_{i}^{n}\right\|_{\mathcal{M}}\right\}$ is bounded which implies that $\left\|\mathcal{E} w_{i-1}^{n}-w_{i}^{n}\right\|_{\mathcal{M}} \rightarrow 0$ since $\alpha_{k-i}^{n} \rightarrow \infty$ as $n \rightarrow \infty$ and hence,

$$
\left\|\mathcal{E} w_{i-1}^{*}-w_{i}^{*}\right\|_{\mathcal{M}} \leq \liminf _{n \rightarrow \infty}\left\|\mathcal{E} w_{i-1}^{n}-w_{i}^{n}\right\|_{\mathcal{M}}=0 .
$$

Together,

$$
\begin{aligned}
\operatorname{TGV}_{\alpha^{*}}^{k, l}(u) \leq \sum_{i=1}^{k} \alpha_{k-i}^{*}\left\|\mathcal{E} w_{i-1}^{*}-w_{i}^{*}\right\|_{\mathcal{M}} \leq \sum_{i=1}^{k} \liminf _{n \rightarrow \infty} \alpha_{k-i}^{n} \| \mathcal{E} w_{i-1}^{n} & -w_{i}^{n} \|_{\mathcal{M}} \\
& \leq \liminf _{n \rightarrow \infty} \operatorname{TGV}_{\alpha^{n}}^{k, l}\left(u^{n}\right)
\end{aligned}
$$

which shows the addition.

Combining this with the estimate on the limes superior gives the stated convergence for $\operatorname{TGV}_{\alpha^{n}}^{k, l}(u)$. In particular, this includes the case $\operatorname{TGV}_{\alpha^{*}}^{k, l}(u)=\infty$. 
Theorem 4.8. Let $1 \leq p \leq \frac{d}{d-1}$ with $p<\infty, q \in[1, \infty[$ and the operator $K$ : $L^{p}\left(\Omega, \operatorname{Sym}^{l}\left(\mathbb{R}^{d}\right)\right) \rightarrow Y$ be linear and continuous in some normed space $Y$. Take $f^{\dagger} \in Y$ and $\left\{f^{n}\right\}$ to be a sequence in $Y$ which satisfies $\left\|f^{n}-f^{\dagger}\right\|_{Y} \leq \delta_{n}$ for a monotonically decreasing null sequence $\left\{\delta_{n}\right\}$ of positive real numbers. Further take a sequence of positive weights $\left\{\alpha^{n}\right\}$ and, denoting by

$$
\beta_{n}=\min \left\{\alpha_{i}^{n} \mid 0 \leq i \leq k-1\right\}, \quad m=\max \left\{m^{\prime} \mid \lim _{n \rightarrow \infty} \frac{1}{\beta_{n}} \alpha_{k-i}^{n}=\infty \text { for all } i<m^{\prime}\right\}
$$

suppose that

1. $\beta_{n} \rightarrow 0$ and $\frac{\delta_{n}^{q}}{\beta_{n}} \rightarrow 0$ as $n \rightarrow \infty$,

2. there is a $u^{\dagger} \in \operatorname{BD}^{m}\left(\Omega, \operatorname{Sym}^{l}\left(\mathbb{R}^{d}\right)\right)$ such that $f^{\dagger}=K u^{\dagger}$.

Then, there exists a sequence of solutions $\left\{u^{n}\right\}$ of (4.2) with data $f^{n}$ and weights $\alpha^{n}$ possessing a $\mathrm{BD}\left(\Omega, \operatorname{Sym}^{l}\left(\mathbb{R}^{d}\right)\right)$-weakly* convergent subsequence $\left\{u^{n_{\nu}}\right\}$ with limit $u^{*}$ such that

1. $u^{*}$ is a $\mathrm{TGV}_{\alpha^{*}}^{k, l}$-minimizing solution of $K u=f^{\dagger}$ for some $\alpha^{*} \in[1, \infty]^{k}$ and

2. $\lim _{\nu \rightarrow \infty} \frac{1}{\beta_{n_{\nu}}} \mathrm{TGV}_{\alpha^{n_{\nu}}}^{k, l}\left(u^{n_{\nu}}\right)=\mathrm{TGV}_{\alpha^{*}}^{k, l}\left(u^{*}\right)$.

Proof. At first note that, as shown in Corollary 4.3, there always exists a solution $u^{n} \in X^{\perp}$ of (4.2) with data $f^{n}$ and weights $\alpha^{n}$ (where $X^{\perp}$ is given by (4.3)). We denote by $\left\{u^{n}\right\}$ a sequence of such solutions.

Then we define $\left.\tilde{\alpha}^{n}=\frac{\alpha^{n}}{\beta_{n}} \in\right] 0, \infty\left[^{k}\right.$. For the $i$ for which $\lim _{n \rightarrow \infty} \tilde{\alpha}_{k-i}^{n}=\infty$ we set $\alpha_{i}^{*}=\infty$ while for the remaining $i$, an accumulation point $\alpha_{i}^{*} \in\left[1, \infty\left[\right.\right.$ of $\left\{\tilde{\alpha}_{k-i}^{n}\right\}$ can be found. This gives a $\alpha^{*} \in[1, \infty]^{k}$ and a subsequence $\left\{\alpha^{n_{\nu}}\right\}$ such that $\tilde{\alpha}^{n_{\nu}} \rightarrow \alpha^{*}$ as $\nu \rightarrow \infty$. From now on, we will consider $\left\{u^{n_{\nu}}\right\}$, the corresponding subsequence of solutions.

Now let $u^{\dagger} \in \operatorname{BD}^{m}\left(\Omega, \operatorname{Sym}^{l}\left(\mathbb{R}^{d}\right)\right)$ such that $K u^{\dagger}=f^{\dagger}$ which exists by assumption. Then, $\operatorname{TGV}_{\alpha^{n_{\nu}}}^{k, l}\left(u^{\dagger}\right)$ is a null-sequence: Indeed, first note that $\operatorname{TGV}_{\alpha^{n_{\nu}}}^{k, l}\left(u^{\dagger}\right)=\beta_{n_{\nu}} \operatorname{TGV}_{\tilde{\alpha}^{n_{\nu}}}^{k, l}\left(u^{\dagger}\right)$ according to the primal representation (3.3), for instance. Proposition 4.7 then implies

$$
\lim _{\nu \rightarrow \infty} \operatorname{TGV}_{\tilde{\alpha}^{n_{\nu}}}^{k, l}\left(u^{\dagger}\right)=\operatorname{TGV}_{\alpha^{*}}^{k, l}\left(u^{\dagger}\right)
$$

for which the right-hand side is finite which can be seen by (4.4) plugging $w_{i}^{\nu}=\mathcal{E}^{i} u^{\dagger}$ for $0 \leq i<m, w_{i}^{\nu}=0$ for $m \leq i \leq k$ into the corresponding minimization problem. Consequently, $\mathrm{TGV}_{\alpha^{n_{\nu}}}^{k, l}\left(u^{\dagger}\right) \rightarrow 0$ as $\nu \rightarrow \infty$. With that, we get

$$
\frac{\left\|K u^{n_{\nu}}-f^{n_{\nu}}\right\|_{Y}^{q}}{q}+\mathrm{TGV}_{\alpha^{n_{\nu}}}^{k, l}\left(u^{n_{\nu}}\right) \leq \frac{\delta_{n_{\nu}}^{q}}{q}+\mathrm{TGV}_{\alpha^{n_{\nu}}}^{k, l}\left(u^{\dagger}\right) \rightarrow 0 \quad \text { as } \quad \nu \rightarrow \infty
$$

and hence, $K u^{n_{\nu}} \rightarrow f^{\dagger}$ in $Y$. Also, dividing the inequality in (4.5) by $\beta_{n_{\nu}}$ leads to

$$
\mathrm{TGV}_{\tilde{\alpha}^{n_{\nu}}}^{k, l}\left(u^{n_{\nu}}\right) \leq \frac{\left\|K u^{n_{\nu}}-f^{n_{\nu}}\right\|_{Y}^{q}}{\beta_{n_{\nu}} q}+\mathrm{TGV}_{\tilde{\alpha}^{n_{\nu}}}^{k, l}\left(u^{n_{\nu}}\right) \leq \frac{\delta_{n_{\nu}}^{q}}{\beta_{n_{\nu}} q}+\mathrm{TGV}_{\tilde{\alpha}^{n_{\nu}}}^{k, l}\left(u^{\dagger}\right)
$$


for which the assumption on $\left\{\beta_{n}\right\}$ yields

$$
\limsup _{\nu \rightarrow \infty} \operatorname{TGV}_{\tilde{\alpha}^{n_{\nu}}}^{k, l}\left(u^{n_{\nu}}\right) \leq \limsup _{\nu \rightarrow \infty}\left(\frac{\delta_{n_{\nu}}^{q}}{\beta_{n_{\nu}} q}+\operatorname{TGV}_{\tilde{\alpha}^{n_{\nu}}}^{k, l}\left(u^{\dagger}\right)\right)=\operatorname{TGV}_{\alpha^{*}}^{k, l}\left(u^{\dagger}\right) .
$$

The next step is to bound $\left\{u^{n_{\nu}}\right\}$ in terms of the $\operatorname{BD}\left(\Omega, \operatorname{Sym}^{l}\left(\mathbb{R}^{d}\right)\right)$-norm. For this purpose, let $R: L^{d /(d-1)}\left(\Omega, \operatorname{Sym}^{l}\left(\mathbb{R}^{d}\right)\right) \rightarrow \operatorname{ker}\left(\mathcal{E}^{k}\right)$ be a linear, continuous, onto projection. Denoting $\mathbf{1}=(1, \ldots, 1) \in \mathbb{R}^{k}$, we get, by Proposition 3.11 and monotonicity with respect to the weights (Remark 3.8)

$$
\left\|u^{n_{\nu}}-R u^{n_{\nu}}\right\|_{d /(d-1)} \leq C \operatorname{TGV}_{1}^{k, l}\left(u^{n_{\nu}}\right) \leq C \operatorname{TGV}_{\tilde{\alpha}^{n_{\nu}}}^{k, l}\left(u^{n_{\nu}}\right)
$$

and hence boundedness of $\left\|u^{n_{\nu}}-R u^{n_{\nu}}\right\|_{d /(d-1)}$. Similar as in the proof of Corollary 4.3, injectivity of $K$ on $X^{\perp}$ implies the existence of a constant such that $\left\|R u^{n_{\nu}}\right\|_{d /(d-1)} \leq$ $C\left\|K R u^{n_{\nu}}\right\|_{Y}$ and, consequently, for $\tilde{C}>0$ sufficiently large,

$C^{-1}\left\|R u^{n_{\nu}}\right\|_{d /(d-1)}-\tilde{C} \leq\left\|K R u^{n_{\nu}}\right\|_{Y}-\|K\|\left\|u^{n_{\nu}}-R u^{n_{\nu}}\right\|_{d /(d-1)}-\left\|f^{n_{\nu}}\right\|_{Y} \leq\left\|K u^{n_{\nu}}-f^{n_{\nu}}\right\|_{Y}$

with right hand side being bounded. Thus also $\left\{\left\|R u^{n_{\nu}}\right\|_{d /(d-1)}\right\}$ - and hence $\left\{\left\|u^{n_{\nu}}\right\|_{d /(d-1)}\right\}$ - is bounded. In order to obtain a bound on $\left\{\left\|\mathcal{E} u^{n_{\nu}}\right\|_{\mathcal{M}}\right\}$, we use Proposition 3.11 and monotonicity again to estimate

$$
\left\|\mathcal{E} u^{n_{\nu}}\right\|_{\mathcal{M}} \leq C\left(\left\|u^{n_{\nu}}\right\|_{1}+\operatorname{TGV}_{1}^{k, l}\left(u^{n_{\nu}}\right)\right) \leq C\left\|u^{n_{\nu}}\right\|_{1}+C \operatorname{TGV}_{\tilde{\alpha}^{n_{\nu}}}^{k, l}\left(u^{n_{\nu}}\right)
$$

where the last term is bounded again due to (4.6) and the boundedness of $\left\|u^{n_{\nu}}\right\|_{d /(d-1)}$.

Thus $\left\{u^{n_{\nu}}\right\}$ is bounded in $\operatorname{BD}\left(\Omega, \operatorname{Sym}^{l}\left(\mathbb{R}^{d}\right)\right)$ and possesses a subsequence (not relabeled) weakly* converging to some $u^{*} \in \mathrm{BD}\left(\Omega, \operatorname{Sym}^{l}\left(\mathbb{R}^{d}\right)\right)$. By virtue of the embedding result of Theorem 2.3, $u^{n_{\nu}} \rightarrow u^{*}$ also in $L^{p}\left(\Omega, \operatorname{Sym}^{l}\left(\mathbb{R}^{d}\right)\right)$ and since $K$ is weakly sequentially continuous, $K u^{n_{\nu}} \rightarrow K u^{*}$. As $K u^{n_{\nu}} \rightarrow f^{\dagger}$, it follows that $K u^{*}=f^{\dagger}$.

To show the convergence of $\left\{\operatorname{TGV}_{\alpha^{n_{\nu}}}^{k, l}\left(u^{n_{\nu}}\right)\right\}$, observe that the addition in Proposition 4.7 gives

$$
\operatorname{TGV}_{\alpha^{*}}^{k, l}\left(u^{*}\right) \leq \liminf _{\nu \rightarrow \infty} \operatorname{TGV}_{\tilde{\alpha}^{n_{\nu}}}^{k, l}\left(u^{n_{\nu}}\right)
$$

which in turn implies

$$
\operatorname{TGV}_{\alpha^{*}}^{k, l}\left(u^{*}\right) \leq \liminf _{\nu \rightarrow \infty} \operatorname{TGV}_{\tilde{\alpha}^{n_{\nu}}}^{k, l}\left(u^{n_{\nu}}\right) \leq \limsup _{\nu \rightarrow \infty} \operatorname{TGV}_{\tilde{\alpha}^{n_{\nu}}}^{k, l}\left(u^{n_{\nu}}\right) \leq \operatorname{TGV}_{\alpha^{*}}^{k, l}\left(u^{\dagger}\right)
$$

Now since $u^{\dagger}$ is arbitrary in $\operatorname{BD}^{m}\left(\Omega, \operatorname{Sym}^{l}\left(\mathbb{R}^{d}\right)\right)$ such that $K u^{\dagger}=f^{\dagger}$, and since $\operatorname{TGV}_{\alpha^{*}}^{k, l}(u)=$ $\infty$ for any $u \in L^{p}\left(\Omega, \operatorname{Sym}^{l}\left(\mathbb{R}^{d}\right)\right) \backslash \operatorname{BD}^{m}\left(\Omega, \operatorname{Sym}^{l}\left(\mathbb{R}^{d}\right)\right.$ ) (according to (4.4) and noting that $\alpha_{k-i}^{*}=\infty$ for $1 \leq i<m$ ) we get that

$$
\operatorname{TGV}_{\alpha^{*}}^{k, l}\left(u^{*}\right) \leq \operatorname{TGV}_{\alpha^{*}}^{k, l}(u) \text { for any } u \in L^{p}\left(\Omega, \operatorname{Sym}^{l}\left(\mathbb{R}^{d}\right)\right) \text { such that } K u=f^{\dagger},
$$

meaning that $u^{*}$ is a $\mathrm{TGV}_{\alpha^{*}}^{k, l}$-minimizing solution of $K u=f^{\dagger}$. Considering $u^{*}$ in the estimate on the limes superior (4.6) then gives $\operatorname{TGV}_{\tilde{\alpha}^{n_{\nu}}}^{k, l}\left(u^{n_{\nu}}\right) \rightarrow \operatorname{TGV}_{\alpha^{*}}^{k, l}\left(u^{*}\right)$ as $\nu \rightarrow \infty$. 
One can think of many refinements and extensions of the above theorem.

Remark 4.9.

1. For any sequence $\left\{u^{n}\right\}$ of solutions which is bounded in $L^{1}\left(\Omega, \operatorname{Sym}^{l}\left(\mathbb{R}^{d}\right)\right)$ there already exists a subsequence with the properties stated in Theorem 4.8.

This is because then, for a subsequence where $\left\{\tilde{\alpha}^{n_{\nu}}\right\}$ converges, the corresponding $\left\{\mathcal{E} u^{n_{\nu}}\right\}$ is already bounded (see (4.7)), leading to the desired boundedness in $\operatorname{BD}\left(\Omega, \operatorname{Sym}^{l}\left(\mathbb{R}^{d}\right)\right.$ ).

2. If $\left\{\frac{1}{\beta_{n}} \alpha_{k-m}^{n}\right\}$ is bounded for some $m$ such that there is a $u^{\dagger} \in \operatorname{BD}^{m}\left(\Omega, \operatorname{Sym}^{l}\left(\mathbb{R}^{d}\right)\right)$ with $K u^{\dagger}=f^{\dagger}$, then $\mathrm{TGV}_{\alpha^{n}}^{k, l}\left(u^{\dagger}\right) \rightarrow 0$ and $K u^{n} \rightarrow f^{\dagger}$ as $n \rightarrow \infty$, i.e., for the whole sequence.

In this case, we can extract from each subsequence of $\left\{u^{n}\right\}$ another subsequence $\left\{u^{n_{\nu}}\right\}$ such that $\lim _{\nu \rightarrow \infty} \tilde{\alpha}^{n_{\nu}}=\alpha^{*}$ for some $\alpha^{*}$ with $\alpha_{k-m}^{*}<\infty$. As $u^{\dagger} \in \operatorname{BD}^{m}\left(\Omega, \operatorname{Sym}^{l}\left(\mathbb{R}^{d}\right)\right)$ we have $\lim _{\nu \rightarrow \infty} \operatorname{TGV}_{\tilde{\alpha}^{n_{\nu}}}^{k, l}\left(u^{\dagger}\right)=\mathrm{TGV}_{\alpha^{*}}^{k, l}\left(u^{\dagger}\right)<\infty$ again by Proposition 4.7. It follows that $\mathrm{TGV}_{\alpha^{n_{\nu}}}^{k, l}\left(u^{\dagger}\right) \rightarrow 0$ and $K u^{n_{\nu}} \rightarrow f^{\dagger}$ as $\nu \rightarrow \infty$. As the initial subsequence was arbitrary, the convergence also holds for the whole sequence.

3. If the whole sequence $\left\{\tilde{\alpha}^{n}\right\}$ converges to some $\alpha^{*}$, then each accumulation point of $\left\{u^{n}\right\}$ is a $\mathrm{TGV}_{\alpha^{*}}^{k, l}$-minimizing solution of $K u=f^{\dagger}$. In case the latter is unique, we get weak*-convergence of the whole sequence $\left\{u^{n}\right\}$.

4. In view of the conditions $\beta_{n} \rightarrow 0$ and $\frac{\delta_{n}^{q}}{\beta_{n}} \rightarrow 0$ one might interpret $\left\{\beta_{n}\right\}$ as the "regularization parameter" and $\left\{\tilde{\alpha}^{n}\right\}$ as fine-tuning parameters.

Nevertheless, it is also possible to formulate conditions on $\left\{\alpha^{n}\right\}$ which lead to the same conclusion without introducing $\left\{\beta_{n}\right\}$ : For instance, $\alpha_{k-m^{\prime}}^{n} \rightarrow 0$ for a $1 \leq m^{\prime} \leq k, \frac{\delta_{n}^{q}}{\alpha_{i}^{n}} \rightarrow 0$ for each $1 \leq i \leq k$ and the existence of $u^{\dagger} \in \operatorname{BD}^{k}\left(\Omega, \operatorname{Sym}^{l}\left(\mathbb{R}^{d}\right)\right)$ such that $K u^{\dagger}=f^{\dagger}$ is sufficient.

In order to see this, note that we immediately have $\beta_{n} \rightarrow 0$. Suppose that there exists a $c>0$ and an infinite set $N \subset \mathbb{N}$ such that $\frac{\delta_{n}^{q}}{\beta_{n}} \geq c$ for all $n \in N$. Then, since the minimum is taken over finitely many elements, there is an $i$ such that $\beta_{n}=$ $\alpha_{i}^{n}$ for infinitely many $n \in N$. Consequently, $\frac{\delta_{n}^{q}}{\alpha_{i}^{n}} \geq c$ for infinitely many $n$ which contradicts $\lim _{n \rightarrow \infty} \frac{\delta_{n}^{q}}{\alpha_{i}^{n}}=0$. This establishes $\frac{\delta_{n}^{q}}{\beta_{n}} \rightarrow 0$ as $n \rightarrow \infty$. For the above $i$, we also have that $\left\{\frac{1}{\beta_{n}} \alpha_{i}^{n}\right\}$ does not tend to infinity, so $m \leq k-i$ and, consequently, $\operatorname{BD}^{m}\left(\Omega, \operatorname{Sym}^{l}\left(\mathbb{R}^{d}\right)\right)$. This also shows that we are in general not able to determine $m$ without further assumptions on $\left\{\alpha^{n}\right\}$.

These conditions on $\left\{\alpha^{n}\right\}$ are clear stronger than the ones stated in Theorem 4.8. This applies in particular for the regularity assumption $u^{\dagger} \in \operatorname{BD}^{k}\left(\Omega, \operatorname{Sym}^{l}\left(\mathbb{R}^{d}\right)\right)$. In fact, the $m$ introduced in the theorem is the smallest $m$ for which the argumentation carries through under the assumption $u^{\dagger} \in \operatorname{BD}^{m}\left(\Omega, \operatorname{Sym}^{l}\left(\mathbb{R}^{d}\right)\right)$. 
5. If $\alpha^{*}$ is finite, then for the subsequence $\left\{u^{n_{\nu}}\right\}$ obtained in the theorem it also holds that $\mathrm{TGV}_{\alpha^{*}}^{k, l}\left(u^{n_{\nu}}\right) \rightarrow \mathrm{TGV}_{\alpha^{*}}^{k, l}\left(u^{*}\right)$ as $\nu \rightarrow \infty$.

By lower semi-continuity of $\mathrm{TGV}_{\alpha^{*}}^{k, l}$, we only need to establish an estimate for the limes superior. For this purpose, denote by $w_{0}^{\nu}, \ldots, w_{k}^{\nu}$ the feasible symmetric tensor fields which establish the minimum in the primal representation (4.4) for $\operatorname{TGV}_{\alpha^{n_{\nu}}}^{k, l}\left(u^{n_{\nu}}\right)$. Obviously, these also realize $\operatorname{TGV}_{\tilde{\alpha}^{n_{\nu}}}^{k, l}\left(u^{n_{\nu}}\right)$. Hence,

$$
\begin{aligned}
\operatorname{TGV}_{\alpha^{*}}^{k, l}\left(u^{n_{\nu}}\right) \leq \sum_{i=1}^{k} \alpha_{k-i}^{*}\left\|\mathcal{E} w_{i-1}^{\nu}-w_{i}^{\nu}\right\|_{\mathcal{M}} \leq c_{\nu} \sum_{i=1}^{k} \tilde{\alpha}_{k-i}^{n_{\nu}} \| \mathcal{E} w_{i-1}^{\nu} & -w_{i}^{\nu} \|_{\mathcal{M}} \\
& =\frac{c_{\nu}}{\beta_{n_{\nu}}} \operatorname{TGV}_{\alpha^{n_{\nu}}}^{k, l}\left(u^{n_{\nu}}\right)
\end{aligned}
$$

where $c_{\nu}=\max _{i=0, \ldots, k-1} \alpha_{i}^{*} / \tilde{\alpha}_{i}^{n_{\nu}}$ which is finite by assumption. The right-hand side then converges appropriately, giving $\lim \sup _{\nu \rightarrow \infty} \mathrm{TGV}_{\alpha^{*}}^{k, l}\left(u^{n_{\nu}}\right) \leq \mathrm{TGV}_{\alpha^{*}}^{k, l}\left(u^{*}\right)$.

6. If the ratios $\left\{\frac{\alpha_{i}^{n}}{\alpha_{i-1}^{n}}\right\}$ are bounded from above and below away from 0 for $i=1, \ldots, k-1$, then $\left\{\tilde{\alpha}^{n}\right\}$ is also bounded and hence, each accumulation point $\alpha^{*}$ will always be finite. If these ratios converge and the limit is strictly positive, then accumulation points are unique up to a positive common factor. The latter follows from $\frac{\alpha_{i}^{*}}{\alpha_{i-1}^{*}}=\lim _{n \rightarrow \infty} \frac{\alpha_{i}^{n}}{\alpha_{i-1}^{n}}$ meaning that the ratios of adjacent entries of $\alpha^{*}$ are independent from each subsequence of $\left\{\tilde{\alpha}^{n}\right\}$. As a consequence, the set of $\mathrm{TGV}_{\alpha^{*}}^{k, l}$-minimizing solutions is independent of the accumulation point $\alpha^{*}$.

Collecting some of these results finally gives:

Corollary 4.10. If, in the situation of Theorem 4.8, the items 1. and 2. are replaced by 1. $\alpha_{i}^{n} \rightarrow 0$ and $\frac{\delta_{n}^{q}}{\alpha_{i}^{n}} \rightarrow 0$ as $n \rightarrow \infty$ for some $i=0, \ldots, k-1$,

2. $\left\{\frac{\alpha_{i}^{n}}{\alpha_{i-1}^{n}}\right\}$ converges to some positive value for each $i=1, \ldots, k-1$,

3. there exists a $u^{\dagger} \in \mathrm{BD}\left(\Omega, \operatorname{Sym}^{l}\left(\mathbb{R}^{d}\right)\right)$ such that $K u^{\dagger}=f^{\dagger}$,

then it holds that there exists a $\left.\alpha^{*} \in\right] 0, \infty\left[^{k}\right.$ and a subsequence $\left\{u^{n_{\nu}}\right\}$ weakly* converging to some minimum- $\mathrm{TGV}_{\alpha^{*}}^{k, l}$ solution $u^{*}$ in $\operatorname{BD}\left(\Omega, \operatorname{Sym}^{l}\left(\mathbb{R}^{d}\right)\right)$ such that

$$
K u^{n} \rightarrow f^{\dagger} \quad \text { and } \quad \operatorname{TGV}_{\alpha^{*}}^{k, l}\left(u^{n}\right) \rightarrow \mathrm{TGV}_{\alpha^{*}}^{k, l}\left(u^{*}\right) \quad \text { as } \quad n \rightarrow \infty
$$

for the whole sequence.

If the minimum-TGV $\alpha_{\alpha^{*}}^{k, l}$ solution is unique, then $u^{n} \stackrel{*}{\rightarrow} u^{*}$ as $n \rightarrow \infty$.

Proof. The only point which does not directly follow from Remark 4.9 is the convergence of $\left\{\operatorname{TGV}_{\alpha^{*}}^{k, l}\left(u^{n}\right)\right\}$ : For an arbitrary subsequence of $\left\{u^{n_{\nu}}\right\}$ we can choose another subsequence 
(not relabeled) such that $\left\{\alpha^{n_{\nu}}\right\} \rightarrow \alpha^{* *}$ and $u^{n_{\nu}} \stackrel{*}{\rightarrow} u^{* *}$ as $\nu \rightarrow \infty$ with $u^{* *}$ being a minimum-TGV $\alpha^{k *}$ solution. From Remark 4.9 we know that $\alpha^{*}=c \alpha^{* *}$ for some $c>0$ and that $u^{* *}$ is also a minimum- $\mathrm{TGV}_{\alpha^{*}}^{k, l}$ solution. Hence, as $\alpha^{* *}$ is finite,

$$
\operatorname{TGV}_{\alpha^{*}}\left(u^{n_{\nu}}\right)=c \operatorname{TGV}_{\alpha^{* *}}\left(u^{n_{\nu}}\right) \rightarrow c \operatorname{TGV}_{\alpha^{* *}}^{k, l}\left(u^{* *}\right)=\operatorname{TGV}_{\alpha^{*}}^{k, l}\left(u^{* *}\right)=\operatorname{TGV}_{\alpha^{*}}^{k, l}\left(u^{*}\right)
$$

as $\nu \rightarrow \infty$. This shows the desired convergence statement.

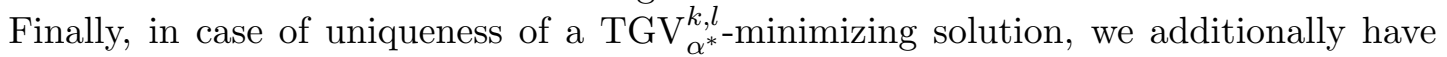
$u^{* *}=u^{*}$ in the above argumentation, giving $u^{n} \stackrel{*}{\rightarrow} u^{*}$ as $n \rightarrow \infty$.

In particular, in view of of this corollary, the situation for multiparameter regularization with TGV is, in special cases, similar to the one-parameter case: Letting noise and parameters tend to zero appropriately then yields solutions which converge to a minimal$\mathrm{TGV}_{\alpha^{*}}^{k, l}$-solution with convergence in terms of $\mathrm{TGV}_{\alpha^{*}}^{k, l}$. This is the motivation for studying this kind of strict convergence in the next section (see also Theorem 4.5).

\section{$5 \quad \mathrm{TGV}_{\alpha}^{k, l}$-strict convergence}

Definition 5.1. Let $k, l \in \mathbb{N}, k \geq 1, l \geq 0$ and $\alpha \in] 0, \infty\left[^{k}\right.$. We say that the sequence $\left\{u^{n}\right\}$ in $\operatorname{BD}\left(\Omega, \operatorname{Sym}^{l}\left(\mathbb{R}^{d}\right)\right)$ converges strictly with respect to $\mathrm{TGV}_{\alpha}^{k, l}$ or $\mathrm{TGV}_{\alpha}^{k, l}$-strictly to an $u \in \operatorname{BD}\left(\Omega, \operatorname{Sym}^{l}\left(\mathbb{R}^{d}\right)\right)$ if

$$
\left\|u^{n}-u\right\|_{1} \rightarrow 0 \quad \text { and } \quad \operatorname{TGV}_{\alpha}^{k, l}\left(u^{n}\right) \rightarrow \operatorname{TGV}_{\alpha}^{k, l}(u) \text { as } n \rightarrow \infty .
$$

In this case, we also write $u^{n} \stackrel{\mathrm{TGV}_{\alpha}^{k, l}}{\longrightarrow} u$ as $n \rightarrow \infty$ in $\operatorname{BD}\left(\Omega, \operatorname{Sym}^{l}\left(\mathbb{R}^{d}\right)\right)$.

Some properties of this generalization of strict convergence in $\operatorname{BD}\left(\Omega, \operatorname{Sym}^{l}\left(\mathbb{R}^{d}\right)\right)$ follow immediately.

Remark 5.2. Strict convergence with respect to $\mathrm{TGV}_{\alpha}^{k, l}$ can equivalently be defined by

$$
u^{n} \stackrel{*}{\rightarrow} u \text { in } \operatorname{BD}\left(\Omega, \operatorname{Sym}^{l}\left(\mathbb{R}^{d}\right)\right) \text { and } \operatorname{TGV}_{\alpha}^{k, l}\left(u^{n}\right) \rightarrow \operatorname{TGV}_{\alpha}^{k, l}(u) \quad \text { as } \quad n \rightarrow \infty \text {. }
$$

This is because by the compact embedding $\operatorname{BD}\left(\Omega, \operatorname{Sym}^{l}\left(\mathbb{R}^{d}\right)\right) \hookrightarrow L^{1}\left(\Omega, \operatorname{Sym}^{l}\left(\mathbb{R}^{d}\right)\right.$ ) (see Theorem 2.3), $u^{n} \stackrel{*}{\rightarrow} u$ implies $\left\|u^{n}-u\right\|_{1} \rightarrow 0$. On the other hand, if $\left\|u^{n}-u\right\|_{1} \rightarrow 0$ and $\operatorname{TGV}_{\alpha}^{k, l}\left(u^{n}\right) \rightarrow \operatorname{TGV}_{\alpha}^{k, l}(u)$, then $\left\{u^{n}\right\}$ is bounded in $\operatorname{BD}\left(\Omega, \operatorname{Sym}^{l}\left(\mathbb{R}^{d}\right)\right.$ ) (according to the norm equivalence in Corollary 3.13) and admits a weakly* convergent subsequence with limit $u^{*}$ which has to coincide with $u$, again by compact embedding. The usual subsequence argument then shows weak ${ }^{*}$-convergence of the whole sequence, i.e. $u^{n} \stackrel{*}{\rightarrow} u$ in $\operatorname{BD}\left(\Omega, \operatorname{Sym}^{l}\left(\mathbb{R}^{d}\right)\right)$ as $n \rightarrow \infty$. 
Remark 5.3. From the fact that strong convergence in $\operatorname{BD}\left(\Omega, \operatorname{Sym}^{l}\left(\mathbb{R}^{d}\right)\right)$ implies convergence of the corresponding $\operatorname{TGV}_{\alpha}^{k, l}$ values (as $\operatorname{TGV}_{\alpha}^{k, l}$ is continuous in $\operatorname{BGV}^{k}\left(\Omega, \operatorname{Sym}^{l}\left(\mathbb{R}^{d}\right)\right)=$ $\operatorname{BD}\left(\Omega, \operatorname{Sym}^{l}\left(\mathbb{R}^{d}\right)\right)$, see Corollary 3.13) and the alternative definition of $\mathrm{TGV}_{\alpha}^{k, l}$-strict convergence in Remark 5.2 we obtain the following implications for an arbitrary sequence $\left\{u^{n}\right\}$ and $u$ in $\operatorname{BD}\left(\Omega, \operatorname{Sym}^{l}\left(\mathbb{R}^{d}\right)\right)$ :

$$
u^{n} \rightarrow u \quad \Rightarrow \quad u^{n} \stackrel{\mathrm{TGV}_{\alpha}^{k, l}}{\longrightarrow} u \quad \Rightarrow \quad u^{n} \stackrel{*}{\rightarrow} u
$$

as $n \rightarrow \infty$ in $\operatorname{BD}\left(\Omega, \operatorname{Sym}^{l}\left(\mathbb{R}^{d}\right)\right)$. None of the converse implications is true.

The notion of $\mathrm{TGV}_{\alpha}^{k, l}$-strict convergence is thus intermediate between strong and weak* convergence. As for the strict convergence in $\operatorname{BV}(\Omega)$ and $\operatorname{BD}\left(\Omega, \operatorname{Sym}^{l}\left(\mathbb{R}^{d}\right)\right)$, we have that the set of smooth functions is dense with respect to $\mathrm{TGV}_{\alpha}^{k, l}$-strict convergence, a property which fails when strong convergence is regarded. This is a consequence of the following approximation result:

Lemma 5.4. Let $l \geq 0$. For each $u \in \operatorname{BD}\left(\Omega, \operatorname{Sym}^{l}\left(\mathbb{R}^{d}\right)\right)$ and $w \in \operatorname{BD}\left(\Omega, \operatorname{Sym}^{l+1}\left(\mathbb{R}^{d}\right)\right)$ there exists a sequence $\left\{u^{\delta}\right\}, \delta>0$ in $\mathcal{C}^{\infty}\left(\Omega, \operatorname{Sym}^{l}\left(\mathbb{R}^{d}\right)\right) \cap \operatorname{BD}\left(\Omega, \operatorname{Sym}^{l}\left(\mathbb{R}^{d}\right)\right)$ such that

$$
\left\|u^{\delta}-u\right\|_{1} \rightarrow 0 \quad \text { and } \quad\left\|\mathcal{E} u^{\delta}-w\right\|_{\mathcal{M}} \rightarrow\|\mathcal{E} u-w\|_{\mathcal{M}} \quad \text { as } \quad \delta \rightarrow 0 .
$$

Proof. The proof uses techniques from the proofs of [11, Theorem 5.2.2] and [2, Theorem 3.9]. Let $u \in \operatorname{BD}\left(\Omega, \operatorname{Sym}^{l}\left(\mathbb{R}^{d}\right)\right)$ and $w \in \operatorname{BD}\left(\Omega, \operatorname{Sym}^{l+1}\left(\mathbb{R}^{d}\right)\right)$ be fixed. For each $\delta>0$, construct $u^{\delta}$ as follows.

Choose a countable number of sets $\left(\Omega_{h}\right)_{h \in \mathbb{N}}$ such that $\Omega=\bigcup_{h \in \mathbb{N}} \Omega_{h}, \bar{\Omega}_{h} \subset \subset \Omega$ for all $h \in \mathbb{N}$ and any point of $\Omega$ belongs to at most four sets $\Omega_{h}$ (cf. [2, Theorem 3.9] for a construction of such sets). Further, let $\left(\phi_{h}\right)_{h \in \mathbb{N}}$ be a partition of unity relative to $\left(\Omega_{h}\right)_{h \in \mathbb{N}}$, i.e., $\phi_{h} \in \mathcal{C}_{\mathrm{c}}^{\infty}\left(\Omega_{h}\right)$ with $\phi_{h} \geq 0$ for all $h \in \mathbb{N}$ and $\sum_{h \in \mathbb{N}} \phi_{h} \equiv 1$. Finally, let $\rho \in \mathcal{C}_{\mathrm{c}}^{\infty}\left(\mathbb{R}^{d}\right)$ be a standard mollifier, i.e., $\rho$ is radially symmetric, non-negative and satisfies $\int_{\mathbb{R}^{d}} \rho \mathrm{d} x=1$. Denote by $\rho_{\epsilon}$ the function given by $\rho_{\epsilon}(x)=\epsilon^{-d} \rho(x / \epsilon)$.

As $\rho$ is a mollifier, we can find, for any $h \in \mathbb{N}$, an $\epsilon_{h}>0$ such that $\operatorname{supp}\left(\left(u \phi_{h}\right) * \rho_{\epsilon_{h}}\right) \subset$ $\Omega_{h}, \operatorname{supp}\left(\left(w \phi_{h}\right) * \rho_{\epsilon_{h}}\right) \subset \Omega_{h}$ and

$$
\int_{\Omega}\left|\left(u \phi_{h}\right) * \rho_{\epsilon_{h}}-u \phi_{h}\right| \mathrm{d} x<2^{-h} \delta, \quad \int_{\Omega}\left|\left(w \phi_{h}\right) * \rho_{\epsilon_{h}}-w \phi_{h}\right| \mathrm{d} x<2^{-h} \delta
$$

as well as

$$
\int_{\Omega}\left|\left(u \otimes \nabla \phi_{h}\right) * \rho_{\epsilon_{h}}-u \otimes \nabla \phi_{h}\right| \mathrm{d} x<2^{-h} \delta .
$$

Set $u^{\delta}=\sum_{h \in \mathbb{N}}\left(u \phi_{h}\right) * \rho_{\epsilon_{h}}$. This defines the sequence $\left\{u^{\delta}\right\}$ for which we are verifying the claim.

First of all, each $u^{\delta} \in \mathcal{C}^{\infty}\left(\Omega, \operatorname{Sym}^{l}\left(\mathbb{R}^{d}\right)\right)$ as the sums in the definition of $u^{\delta}$ are locally finite. We also immediately see that

$$
\int_{\Omega}\left|u^{\delta}-u\right| \mathrm{d} x \leq \sum_{h \in \mathbb{N}} \int_{\Omega}\left|\left(u \phi_{h}\right) * \rho_{\epsilon_{h}}-u \phi_{h}\right| \mathrm{d} x<\delta
$$


which proves $\left\|u^{\delta}-u\right\|_{1} \rightarrow 0$ as $\delta \rightarrow 0$. Next, let $\varphi \in \mathcal{C}_{\mathrm{c}}^{\infty}\left(\Omega, \operatorname{Sym}^{l+1}\left(\mathbb{R}^{d}\right)\right)$ such that $\|\varphi\|_{\infty} \leq 1$. Testing with $\operatorname{div} \varphi$, dualizing the convolution with $\rho_{\epsilon_{h}}$, using the identities

$$
\begin{aligned}
\phi_{h} \operatorname{div}\left(\varphi * \rho_{\epsilon_{h}}\right) & =\operatorname{div}\left(\phi_{h}\left(\varphi * \rho_{\epsilon_{h}}\right)\right)-\operatorname{tr}\left(\nabla \phi_{h} \otimes\left(\varphi * \rho_{\epsilon_{h}}\right)\right), \\
\operatorname{tr}\left(\nabla \phi_{h} \otimes\left(\varphi * \rho_{\epsilon_{h}}\right)\right) \cdot u & =\operatorname{tr}\left(u \otimes \nabla \phi_{h}\right) \cdot \varphi * \rho_{\epsilon_{h}}
\end{aligned}
$$

and squeezing in $\sum_{h \in \mathbb{N}} u \otimes \nabla \phi_{h}=0$ then leads to

$$
\begin{aligned}
\int_{\Omega} u^{\delta} \cdot \operatorname{div} \varphi \mathrm{d} x & =\sum_{h \in \mathbb{N}} \int_{\Omega}\left(\left(u \phi_{h}\right) * \rho_{\epsilon_{h}}\right) \cdot \operatorname{div} \varphi \mathrm{d} x=\sum_{h \in \mathbb{N}} \int_{\Omega} u \phi_{h} \cdot \operatorname{div}\left(\rho_{\epsilon_{h}} * \varphi\right) \mathrm{d} x \\
& =\sum_{h \in \mathbb{N}} \int_{\Omega} u \cdot \operatorname{div}\left(\phi_{h}\left(\varphi * \rho_{\epsilon_{h}}\right)\right)-u \cdot \operatorname{tr}\left(\nabla \phi_{h} \otimes\left(\varphi * \rho_{\epsilon_{h}}\right)\right) \mathrm{d} x \\
& =\sum_{h \in \mathbb{N}}\left[-\int_{\Omega} \phi_{h}\left(\varphi * \rho_{\epsilon_{h}}\right) \mathrm{d} \mathcal{E} u-\int_{\Omega}\left(\left(u \otimes \nabla \phi_{h}\right) * \rho_{\epsilon_{h}}-u \otimes \nabla \phi_{h}\right) \cdot \varphi \mathrm{d} x\right] .
\end{aligned}
$$

This can be used to estimate, after the adding and subtracting $\sum_{h \in \mathbb{N}}\left(\phi_{h} w\right) * \rho_{\epsilon_{h}}$ and using $\|\varphi\|_{\infty} \leq 1$ as well as $\sup _{x \in \Omega_{h}}\left|\varphi * \rho_{\epsilon_{h}}\right|(x) \leq 1$,

$$
\begin{aligned}
& \int_{\Omega}-u^{\delta} \cdot \operatorname{div} \varphi-w \cdot \varphi \mathrm{d} x=\sum_{h \in \mathbb{N}} {\left[\int_{\Omega} \phi_{h}\left(\varphi * \rho_{\epsilon_{h}}\right) \mathrm{d}\left(\mathcal{E} u-w \mathcal{L}^{d}\right)\right.} \\
&+\int_{\Omega}\left(\left(\phi_{h} w\right) * \rho_{\epsilon_{h}}-\phi_{h} w\right) \cdot \varphi \mathrm{d} x \\
&\left.+\int_{\Omega}\left(\left(u \otimes \nabla \phi_{h}\right) * \rho_{\epsilon_{h}}-u \otimes \nabla \phi_{h}\right) \cdot \varphi \mathrm{d} x\right] \\
& \leq \sum_{h \in \mathbb{N}}\left[\int_{\Omega} \phi_{h} \mathrm{~d}|\mathcal{E} u-w|+2^{-h} \delta+2^{-h} \delta\right] \\
& \leq\|\mathcal{E} u-w\|_{\mathcal{M}}+2 \delta .
\end{aligned}
$$

Taking the supremum over all $\varphi \in \mathcal{C}_{\mathrm{c}}^{\infty}\left(\Omega, \operatorname{Sym}^{l+1}\left(\mathbb{R}^{d}\right)\right)$ with $\|\varphi\|_{\infty} \leq 1$ and letting $\delta \rightarrow 0$ we see that $\lim \sup _{\delta \rightarrow 0}\left\|\mathcal{E} u^{\delta}-w\right\|_{\mathcal{M}} \leq\|\mathcal{E} u-w\|_{\mathcal{M}}$. On the other hand, as $u^{\delta} \rightarrow u$ in $L^{1}\left(\Omega, \operatorname{Sym}^{l}\left(\mathbb{R}^{d}\right)\right)$ as $\delta \rightarrow 0$, we get from lower semi-continuity that

$$
\|\mathcal{E} u-w\|_{\mathcal{M}} \leq \liminf _{\delta \rightarrow 0}\left\|\mathcal{E} u^{\delta}-w\right\|_{\mathcal{M}} \leq\|\mathcal{E} u-w\|_{\mathcal{M}}
$$

Proposition 5.5. Let $k, l \in \mathbb{N}, k \geq 1, l \geq 0$ and $\alpha \in] 0, \infty\left[^{k}\right.$. Then, for each $u \in$ $\mathrm{BD}\left(\Omega, \operatorname{Sym}^{l}\left(\mathbb{R}^{d}\right)\right)$ there exists a sequence $\left\{u^{n}\right\}$ in $\mathcal{C}^{\infty}\left(\Omega, \operatorname{Sym}^{l}\left(\mathbb{R}^{d}\right)\right) \cap \operatorname{BD}\left(\Omega, \operatorname{Sym}^{l}\left(\mathbb{R}^{d}\right)\right)$ such that $\left\{u^{n}\right\}$ converges $\operatorname{TGV}_{\alpha}^{k, l}$-strictly to $u$.

Proof. Let $u \in \operatorname{BD}\left(\Omega, \operatorname{Sym}^{l}\left(\mathbb{R}^{d}\right)\right)$ and choose $w^{*} \in \operatorname{BD}\left(\Omega, \operatorname{Sym}^{l+1}\left(\mathbb{R}^{d}\right)\right)$ such that

$$
\operatorname{TGV}_{\alpha}^{k, l}(u)=\alpha_{k-1}\left\|\mathcal{E} u-w^{*}\right\|_{\mathcal{M}}+\operatorname{TGV}_{\tilde{\alpha}}^{k-1, l+1}\left(w^{*}\right)
$$


for $\tilde{\alpha}=\left(\alpha_{0}, \ldots, \alpha_{k-1}\right)$ agreeing that $\mathrm{TGV}_{()}^{0, l+1}=I_{\{0\}}$. Such a $w^{*}$ exists, see Theorem 3.5. For each $\delta=\frac{1}{n}$ we now set $u^{n}=u^{\delta}$ with $u^{\delta} \in \mathcal{C}^{\infty}\left(\Omega, \operatorname{Sym}^{l}\left(\mathbb{R}^{d}\right)\right) \cap \operatorname{BD}\left(\Omega, \operatorname{Sym}^{l}\left(\mathbb{R}^{d}\right)\right)$ according to the construction in Lemma 5.4. By the minimization characterization (3.3) of $\mathrm{TGV}_{\alpha}^{k, l}$,

$$
\operatorname{TGV}_{\alpha}^{k, l}\left(u^{n}\right) \leq \alpha_{k-1}\left\|\mathcal{E} u^{n}-w^{*}\right\|_{\mathcal{M}}+\mathrm{TGV}_{\tilde{\alpha}}^{k-1, l+1}\left(w^{*}\right)
$$

and as for the sequence $\left\{u^{n}\right\}$ it holds that $\left\|\mathcal{E} u^{n}-w^{*}\right\|_{\mathcal{M}} \rightarrow\left\|\mathcal{E} u-w^{*}\right\|_{\mathcal{M}}$ as $n \rightarrow \infty$, it follows that

$$
\limsup _{n \rightarrow \infty} \operatorname{TGV}_{\alpha}^{k, l}\left(u^{n}\right) \leq \alpha_{k-1}\left\|\mathcal{E} u-w^{*}\right\|_{\mathcal{M}}+\operatorname{TGV}_{\tilde{\alpha}}^{k-1, l+1}\left(w^{*}\right)=\operatorname{TGV}_{\alpha}^{k, l}(u) .
$$

On the other hand, $\lim _{n \rightarrow \infty} u^{n}=u$ in $L^{1}\left(\Omega, \operatorname{Sym}^{l}\left(\mathbb{R}^{d}\right)\right)$, so lower semi-continuity of $\mathrm{TGV}_{\alpha}^{k, l}$ (see Proposition 3.3) gives

$$
\operatorname{TGV}_{\alpha}^{k, l}(u) \leq \liminf _{n \rightarrow \infty} \operatorname{TGV}_{\alpha}^{k, l}\left(u^{n}\right)
$$

which establishes the $\mathrm{TGV}_{\alpha}^{k, l}$-strict convergence to $u$.

Remark 5.6. Note that, given $u \in \operatorname{BD}\left(\Omega, \operatorname{Sym}^{l}\left(\mathbb{R}^{d}\right)\right)$, the construction above yields an approximating sequence $\left\{u^{n}\right\}$ for fixed order $k \in \mathbb{N}$ and weights $\left.\alpha \in\right] 0, \infty\left[{ }^{k}\right.$. Since the elements of this sequence depend on the respective minimizers $w_{1}^{n}, \ldots, w_{k}^{n}$ for (3.3) (see the construction in Lemma 5.4), we cannot assure that $\left\{u^{n}\right\}$ also converges strictly to $u$ with respect to any other choice of $k$ or $\alpha$ (except for the trivial case where $\tilde{\alpha}=\lambda \alpha$ with $\lambda>0$, i.e., if the minimizers remain the same).

Although strong convergence with respect to the norm in $\operatorname{BGV}^{k}\left(\Omega, \operatorname{Sym}^{l}\left(\mathbb{R}^{d}\right)\right)$ for all is equivalent for all weights and orders, the situation is different for $\mathrm{TGV}_{\alpha}^{k, l}$-strict convergence. We show, in the following, counterexamples which demonstrate that for $k \in\{1,2\}$ already convergence notions arise for which neither one is stronger than the other. This justifies in particular a dedicated proof for $\mathrm{TGV}_{\alpha}^{k, l}$-strict density of smooth functions.

Example 5.7. Let $\Omega=] 0,1\left[\right.$ and $\alpha=\left(\alpha_{0}, \alpha_{1}\right)$ with $\alpha_{i}>0$. We construct a sequence $\left\{u^{n}\right\}$ and $u$ in $L^{1}(\Omega)$ such that $\left\{u^{n}\right\}$ converges strictly to $u$ with respect to $\mathrm{TGV}_{\alpha}^{2}$ and does not converge strictly to $u$ with respect to TV, in particular there is a $\delta>0$ and $n_{0} \in \mathbb{N}$ such that $\left|\mathrm{TV}\left(u^{n}\right)-\mathrm{TV}(u)\right|>\delta$ for all $n \geq n_{0}$.

Without loss of generality we can assume $\alpha=(\beta, 1)$. At first we set up the approximating scheme: For any Lipschitz continuous function $u \in \mathcal{C}^{0,1}([0,1])$ (which will be fixed later) we can define

$$
\left.\left.\left.u^{n}(x)=x-\frac{i}{n}+u\left(\frac{i}{n}\right) \quad \text { if } \quad x \in\right] \frac{i}{n}, \frac{i+1}{n}\right] \cap\right] 0,1[
$$

See Figure 1 (a) for an illustration. It follows then by pointwise convergence of $\left\{u^{n}\right\}$ to $u$ 


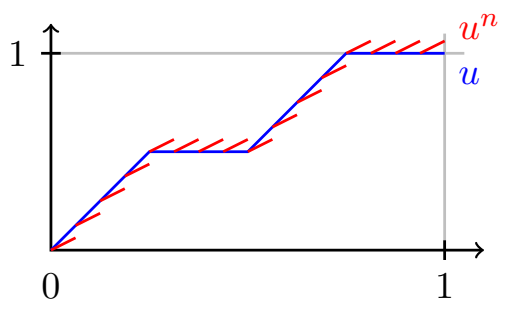

(a)

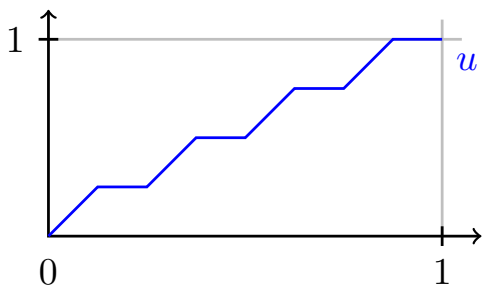

(b)

Figure 1: (a) Illustration of a function $u$ (blue) and its approximation $u^{n}$ (red) for $n=16$ according to (5.1). (b) Illustration of $u$ according to (5.4) for $k=8$.

and Lebesgue's dominated convergence theorem that $\left\|u^{n}-u\right\|_{1} \rightarrow 0$ as $n \rightarrow \infty$. Further, defining $w_{0}(x)=1$ the constant one function, the weak derivative of $u^{n}$ is given by

$$
\left(u^{n}\right)^{\prime}=w_{0}-\sum_{i=1}^{n-1}\left(u\left(\frac{i}{n}\right)-u\left(\frac{i-1}{n}\right)-\frac{1}{n}\right) \delta_{\frac{i}{n}},
$$

and the measure $\delta_{x_{0}}$ is a delta peak at $x_{0}$. Thus, its Radon norm can be given by

$$
\begin{aligned}
\left\|\left(u^{n}\right)^{\prime}\right\|_{\mathcal{M}} & =1+\sum_{i=1}^{n-1}\left|u\left(\frac{i}{n}\right)-u\left(\frac{i-1}{n}\right)-\frac{1}{n}\right|=1+\sum_{i=1}^{n-1} \frac{1}{n}\left|\frac{u\left(\frac{i}{n}\right)-u\left(\frac{i-1}{n}\right)}{n^{-1}}-1\right| \\
& =1+\int_{0}^{1}\left|f^{n}(x)\right| \mathrm{d} x
\end{aligned}
$$

where

$$
f^{n}=\frac{1}{n^{-1}}\left[\sum_{i=1}^{n-1}\left(u\left(\frac{i}{n}\right)-u\left(\frac{i-1}{n}\right)\right) \chi_{\left[\frac{i-1}{n}, \frac{i}{n}[\right.}\right]-w_{0} .
$$

Since $u$ is Lipschitz continuous, $\left\{f^{n}\right\}$ converges to $u^{\prime}-w_{0}$ pointwise almost everywhere. Further, the sequence $\left\{f^{n}\right\}$ is uniformly bounded, thus by Lebesgue's dominated convergence theorem we get

$$
\operatorname{TV}\left(u^{n}\right)=\left\|\left(u^{n}\right)^{\prime}\right\|_{\mathcal{M}}=1+\int_{0}^{1}\left|f^{n}(x)\right| \mathrm{d} x \rightarrow 1+\int_{0}^{1}\left|u^{\prime}(x)-w_{0}(x)\right| \mathrm{d} x
$$

as $n \rightarrow \infty$. For each $w \in \mathrm{BV}(\Omega)$ we now have

$$
\begin{aligned}
\left\|\left(u^{n}\right)^{\prime}-w\right\|_{\mathcal{M}}+\beta\left\|w^{\prime}\right\|_{\mathcal{M}} & =\left\|w_{0}-w\right\|_{1}+\sum_{i=1}^{n-1}\left|u\left(\frac{i}{n}\right)-u\left(\frac{i-1}{n}\right)-\frac{1}{n}\right|+\beta\left\|w^{\prime}\right\|_{\mathcal{M}} \\
& \geq \sum_{i=1}^{n-1}\left|u\left(\frac{i}{n}\right)-u\left(\frac{i-1}{n}\right)-\frac{1}{n}\right|=\left\|\left(u^{n}\right)^{\prime}-w_{0}\right\|_{\mathcal{M}}+\beta\left\|w_{0}^{\prime}\right\|_{\mathcal{M}}
\end{aligned}
$$


which implies that $w_{0}$ is a solution for the minimization problem associated with $\mathrm{TGV}_{\alpha}^{2}$. Thus,

$$
\operatorname{TGV}_{\alpha}^{2}\left(u^{n}\right)=\left\|\left(u^{n}\right)^{\prime}-w_{0}\right\|_{\mathcal{M}} \rightarrow\left\|u^{\prime}-w_{0}\right\|_{1}
$$

as $n \rightarrow \infty$. Now for $k \in \mathbb{N}$ even, sufficiently large such that $\frac{1}{k}<\beta$, define $u \in L^{1}(\Omega)$ such that

$$
u(x)=\left\{\begin{array}{ll}
2 x-\frac{2 i}{k} & \text { if } \left.x \in] \frac{2 i}{k}, \frac{2 i+1}{k}\right], \\
\frac{2 i+2}{k} & \text { if } \left.x \in] \frac{2 i+1}{k}, \frac{2 i+2}{k}\right],
\end{array} \quad 0 \leq i<\frac{k}{2} .\right.
$$

Again we refer to Figure 1 (b) for an illustration. We aim at showing that $w_{0}$ also realizes $\operatorname{TGV}_{\alpha}^{2}(u)$, i.e.,

$$
\operatorname{TGV}_{\alpha}^{2}(u)=\min _{w \in \operatorname{BV}(\Omega)}\left\|u^{\prime}-w\right\|_{1}+\beta\left\|w^{\prime}\right\|_{\mathcal{M}}=\beta\left\|u^{\prime}-w_{0}\right\|_{1}
$$

According to $\left[9\right.$, Section 4.1] this is the case if there exists a $v \in H_{0}^{1}(\Omega)$ such that

$$
\begin{aligned}
-v^{\prime}(x) & \in \operatorname{sgn}\left(u^{\prime}(x)-w_{0}(x)\right) \\
v(x) & \in \beta \operatorname{sgn}\left(w_{0}^{\prime}(x)\right)
\end{aligned}
$$

for almost every $x \in \Omega$ where $\operatorname{sgn}(t)=\{1\}$ if $t>0, \operatorname{sgn}(t)=\{-1\}$ if $t<0$ and $\operatorname{sgn}(t)=$ $[-1,1]$ if $t=0$. One easily verifies that the first condition, $-v^{\prime} \in \operatorname{sgn}\left(u^{\prime}-w_{0}\right)$ is satisfied for

$$
v(x)=\left\{\begin{array}{ll}
-x+\frac{2 i}{k} & \text { if } x \in] \frac{2 i}{k}, \frac{2 i+1}{k}[, \\
x-\frac{2 i+2}{k} & \text { if } x \in] \frac{2 i+1}{k}, \frac{2 i+2}{k}[,
\end{array} \quad 0 \leq i<\frac{k}{2} .\right.
$$

Further we have that $\|v\|_{\infty} \leq \frac{1}{k}<\beta$, implying the second condition, thus $w_{0}$ is a minimizer.

If follows that

$$
\operatorname{TGV}_{\alpha}^{2}(u)=\beta\left\|u^{\prime}-w_{0}\right\|_{1}=\lim _{n \rightarrow \infty} \operatorname{TGV}_{\alpha}^{2}\left(u^{n}\right),
$$

thus $\left\{u^{n}\right\}$ converges strictly with respect to $\mathrm{TGV}_{\alpha}^{2}$ to $u$. But, as $u$ is monotone, $\operatorname{TV}(u)=1$ and (5.3) implies

$$
\left|\mathrm{TV}\left(u^{n}\right)-\mathrm{TV}(u)\right| \rightarrow\left\|u^{\prime}-w_{0}\right\|_{1}>0 \quad \text { as } \quad n \rightarrow \infty .
$$

Hence, $\left\{u^{n}\right\}$ and $u$ have the claimed properties.

Example 5.8. In the same situation as Example 5.7, we can also construct a sequence $\left\{u^{n}\right\}$ and $u$ which converges strictly to $u$ with respect to TV and does not converge strictly with respect to $\mathrm{TGV}_{\alpha}^{2}$ and with the additional property there is a $\delta>0$ and $n_{0} \in \mathbb{N}$ such that $\left|\mathrm{TGV}_{\alpha}^{2}\left(u^{n}\right)-\mathrm{TGV}_{\alpha}^{2}(u)\right|>\delta$ for all $n \geq n_{0}$.

This is done in a similar manner. First, we approximate an arbitrary Lipschitz continuous $u \in \mathcal{C}^{0,1}(\Omega)$ by

$$
\left.\left.\left.u^{n}(x)=u\left(\frac{i}{n}\right) \quad \text { if } \quad x \in\right] \frac{i}{n}, \frac{i+1}{n}\right] \cap\right] 0,1[.
$$


Clearly, $\left\|u^{n}-u\right\|_{1} \rightarrow 0$ as $n \rightarrow \infty$. Moreover, we get that

$$
\left(u^{n}\right)^{\prime}=\sum_{i=1}^{n-1}\left(u\left(\frac{i}{n}\right)-u\left(\frac{i-1}{n}\right)\right) \delta_{\frac{i}{n}}
$$

and, consequently,

$$
\operatorname{TV}\left(u^{n}\right)=\sum_{i=1}^{n-1}\left|u\left(\frac{i}{n}\right)-u\left(\frac{i-1}{n}\right)\right|=\sum_{i=1}^{n-1} \frac{1}{n}\left|\frac{u\left(\frac{i}{n}\right)-u\left(\frac{i-1}{n}\right)}{n^{-1}}\right| \rightarrow \int_{0}^{1}\left|u^{\prime}(x)\right| \mathrm{d} x=\operatorname{TV}(u)
$$

as $n \rightarrow \infty$, meaning TV-strict convergence of $\left\{u^{n}\right\}$ to $u$.

One now sees that $w=0$ then gives as solution to the minimization problem associated with $\operatorname{TGV}_{\alpha}^{2}\left(u^{n}\right)$, hence

$$
\operatorname{TGV}_{\alpha}^{2}\left(u^{n}\right)=\operatorname{TV}\left(u^{n}\right) \rightarrow \operatorname{TV}(u)
$$

as $n \rightarrow \infty$. But choosing $u(x)=x$ for $x \in] 0,1\left[\right.$ implies, as $u \in \operatorname{ker}\left(\mathcal{E}^{2}\right)$, that $\operatorname{TGV}_{\alpha}^{2}(u)=$ $0 \neq \mathrm{TV}(u)$, so the $\mathrm{TGV}_{\alpha}^{2}$-values converge but $\left\{u^{n}\right\}$ does not converge $\mathrm{TGV}_{\alpha}^{2}$-strictly.

Besides the density of smooth functions (Proposition 5.5), there might be more properties for $\mathrm{TGV}_{\alpha}^{k, l}$-strict convergence similar to known results for the classical strict convergence in BV or BD (for instance, the continuity of the trace mapping [5, Theorem 4.14]). However, as the convergence notions are different for each order $k$ and weight $\alpha$, dedicated proofs seems to be necessary for each of these properties. A complete discussion is hence outside the scope of this paper.

\section{Conclusions}

Since well-posedness of Tikhonov-regularization as well as convergence of the regularized solutions for vanishing noise level can be established, we can indeed speak of the TGV functional as a regularizer for linear inverse problems. The functional-analytic framework is given by the spaces $\operatorname{BD}\left(\Omega, \operatorname{Sym}^{l}\left(\mathbb{R}^{d}\right)\right)$ which are topologically equivalent by the spaces induced by the $\operatorname{TGV}_{\alpha}^{k, l}$ semi-norm, i.e., $\operatorname{BGV}^{k}\left(\Omega, \operatorname{Sym}^{l}\left(\mathbb{R}^{d}\right)\right)$. In particular, the prerequisites for respective theorems show that TGV-regularization can essentially be employed whenever it is possible to employ TV or TD-regularization. This holds true, for instance, for a variety of mathematical imaging problems.

Moreover, we see that besides the choice of the appropriate Banach space, the regularization functional itself also has an important qualitative effect on the regularization procedure: Although strong and weak* convergence in $\operatorname{BD}\left(\Omega, \operatorname{Sym}^{l}\left(\mathbb{R}^{d}\right)\right)$ are equivalent, the intermediate notion of $\mathrm{TGV}_{\alpha}^{k, l}$-strict convergence is generally different for different choices of $k$ and $\alpha$. This seems to be related to the qualitative difference of solutions for the Tikhonov-functional minimization problem with $\mathrm{TV}$ and $\mathrm{TGV}_{\alpha}^{2}$ which has been observed in practical applications. 


\section{A Proof of Theorem 2.6}

The result will essentially follows by duality arguments. In the following, let $\Omega \subset \mathbb{R}^{d}$ always be a bounded Lipschitz domain. Furthermore, denote by

$$
\operatorname{ker}(\mathcal{E})^{\perp}=\left\{g \in L^{d}\left(\Omega, \operatorname{Sym}^{k}\left(\mathbb{R}^{d}\right)\right) \mid \int_{\Omega} g \cdot u \mathrm{~d} x=0 \forall u \in \operatorname{ker}(\mathcal{E})\right\} .
$$

which is a closed subspace of $L^{d}\left(\Omega, \operatorname{Sym}^{k}\left(\mathbb{R}^{d}\right)\right)$ since all elements in $\operatorname{ker}(\mathcal{E})$ are polynomials and therefore contained in $L^{\infty}\left(\Omega, \operatorname{Sym}^{k}\left(\mathbb{R}^{d}\right)\right)$. As $\operatorname{ker}(\mathcal{E})$ is finite-dimensional, it is immediate that

$$
L^{d}\left(\Omega, \operatorname{Sym}^{k}\left(\mathbb{R}^{d}\right)\right)=\operatorname{ker}(\mathcal{E}) \oplus \operatorname{ker}(\mathcal{E})^{\perp}
$$

and as both subspaces are closed, the open mapping theorem implies that we can find a continuous projection $R: L^{d}\left(\Omega, \operatorname{Sym}^{k}\left(\mathbb{R}^{d}\right)\right) \rightarrow L^{d}\left(\Omega, \operatorname{Sym}^{k}\left(\mathbb{R}^{d}\right)\right)$ such that $\operatorname{rg}(R)=\operatorname{ker}(\mathcal{E})$ and $\operatorname{ker}(R)=\operatorname{ker}(\mathcal{E})^{\perp}$. The adjoint $R^{*}$ is then a continuous projection in $L^{d /(d-1)}\left(\Omega, \operatorname{Sym}^{k}\left(\mathbb{R}^{d}\right)\right)$ onto $\operatorname{ker}(\mathcal{E})^{\perp \perp}=\operatorname{ker}(\mathcal{E})$ with $\operatorname{kernel} \operatorname{ker}(\mathcal{E})^{\perp}$ and both subspaces interpreted as annihilators in $L^{d /(d-1)}\left(\Omega, \operatorname{Sym}^{k}\left(\mathbb{R}^{d}\right)\right)$.

Lemma A.1. The closure of the set

$$
U=\left\{-\operatorname{div} v \mid v \in \mathcal{C}_{\mathrm{c}}^{\infty}\left(\Omega, \operatorname{Sym}^{k+1}\left(\mathbb{R}^{d}\right)\right),\|v\|_{\infty} \leq 1\right\}
$$

in $L^{d}\left(\Omega, \operatorname{Sym}^{k}\left(\mathbb{R}^{d}\right)\right) \cap \operatorname{ker}(\mathcal{E})^{\perp}$ contains 0 as interior point.

Proof. Let us verify that the functional $F: L^{d /(d-1)}\left(\Omega, \operatorname{Sym}^{k}\left(\mathbb{R}^{d}\right)\right) \rightarrow[0, \infty]$,

$$
F(u)=\|\mathcal{E} u\|_{\mathcal{M}}+I_{\{0\}}\left(R^{*} u\right)
$$

is coercive. For a sequence $\left\{u^{n}\right\}$ in $L^{d /(d-1)}\left(\Omega, \operatorname{Sym}^{k}\left(\mathbb{R}^{d}\right)\right)$ with $\left\|u^{n}\right\|_{d /(d-1)} \rightarrow \infty$ we have, for each $n$, either $F\left(u^{n}\right)=\infty$ or $u^{n} \in \operatorname{BD}\left(\Omega, \operatorname{Sym}^{k}\left(\mathbb{R}^{d}\right)\right) \cap \operatorname{ker}\left(R^{*}\right)$. In the latter case, $R^{*} u^{n}=0$ hence, the Sobolev-Korn-inequality gives

$$
\left\|u^{n}\right\|_{d /(d-1)} \leq C\left\|\mathcal{E} u^{n}\right\|_{\mathcal{M}}=C F\left(u^{n}\right)
$$

for some $C>0$ independent of $n$. Together, this implies $F\left(u^{n}\right) \rightarrow \infty$ resulting in the coercivitiy of $F$.

The Fenchel conjugate $\left.\left.F^{*}: L^{d}\left(\Omega, \operatorname{Sym}^{k}\left(\mathbb{R}^{d}\right)\right) \rightarrow\right]-\infty, \infty\right]$ is consequently continuous at 0 (see [4, Theorem 4.4.10]). As $\operatorname{ker}\left(R^{*}\right)=\operatorname{rg}\left(\mathrm{id}-R^{*}\right)$, this can be computed to be

$$
\begin{aligned}
F^{*}(g) & =\sup _{u \in \operatorname{ker}\left(R^{*}\right)}\langle g, u\rangle-\|\mathcal{E} u\|_{\mathcal{M}}=\sup _{u \in L^{d /(d-1)}\left(\Omega, \operatorname{Sym}^{k}\left(\mathbb{R}^{d}\right)\right)}\left\langle g, u-R^{*} u\right\rangle-\left\|\mathcal{E}\left(u-R^{*} u\right)\right\|_{\mathcal{M}} \\
& =\sup _{u \in L^{d /(d-1)}\left(\Omega, \operatorname{Sym}^{k}\left(\mathbb{R}^{d}\right)\right)}\langle g-R g, u\rangle-\|\mathcal{E}(u)\|_{\mathcal{M}}=I_{U}^{* *}(g-R g) \\
& =I_{\bar{U}}(g-R g) .
\end{aligned}
$$


Continuity in 0 now means that there is an $\varepsilon>0$ such that $B_{\varepsilon}(0) \subset(I-R)^{-1}(\bar{U})$. In particular, for each $g \in L^{d}\left(\Omega, \operatorname{Sym}^{k}\left(\mathbb{R}^{d}\right)\right) \cap \operatorname{ker}(\mathcal{E})^{\perp}$ with $\|g\|_{d}<\varepsilon, g=g-R g \in \bar{U}$ which is what we intended to show.

With this prerequisite, we are able to prove the theorem.

Proof of Theorem 2.6. Let $u \in \mathcal{D}\left(\Omega, \operatorname{Sym}^{k}\left(\mathbb{R}^{d}\right)\right)$ such that $\mathcal{E} u \in \mathcal{M}\left(\Omega, \operatorname{Sym}^{k+1}\left(\mathbb{R}^{d}\right)\right)$ in the distributional sense. Our aim is to show that also $u \in L^{1}\left(\Omega, \operatorname{Sym}^{k}\left(\mathbb{R}^{d}\right)\right)$.

Set $X=L^{d}\left(\Omega, \operatorname{Sym}^{k}\left(\mathbb{R}^{d}\right)\right) \cap \operatorname{ker} \mathcal{E}^{\perp}$ which is, with the induced norm, a Banach space. Choose $\delta>0$ such that

$$
B_{\delta}(0) \subset \overline{\left\{-\operatorname{div} g \mid g \in \mathcal{C}_{\mathrm{c}}^{\infty}\left(\Omega, \operatorname{Sym}^{k+1}\left(\mathbb{R}^{d}\right)\right),\|g\|_{\infty} \leq 1\right\}} \subset X .
$$

Such a $\delta$ exists due to Lemma A.1. We test $u$ with - div $g$ where $g$ is in the set

$$
K_{1}=\left\{g \in \mathcal{C}_{\mathrm{c}}^{\infty}\left(\Omega, \operatorname{Sym}^{k+1}\left(\mathbb{R}^{d}\right)\right) \mid\|g\|_{\infty} \leq \delta^{-1},\|-\operatorname{div} g\|_{d} \leq 1\right\}
$$

which is obviously contained in

$$
K_{2}=\left\{g \in \mathcal{C}_{\mathrm{c}}^{\infty}\left(\Omega, \operatorname{Sym}^{k+1}\left(\mathbb{R}^{d}\right)\right) \mid\|g\|_{\infty} \leq \delta^{-1}\right\} .
$$

Hence, by the fact that $\mathcal{E}(u) \in \mathcal{M}\left(\Omega, \operatorname{Sym}^{k+1}\left(\mathbb{R}^{d}\right)\right)$ and density we get

$$
\sup _{g \in K_{1}}\langle u,-\operatorname{div} g\rangle \leq \sup _{g \in K_{2}}\langle u,-\operatorname{div} g\rangle=\sup _{\substack{g \in \mathcal{C}_{0}\left(\Omega, \operatorname{Sym}^{k+1}\left(\mathbb{R}^{d}\right)\right),\|g\|_{\infty} \leq \delta^{-1}}}\langle\mathcal{E} u, g\rangle=\delta^{-1}\|\mathcal{E} u\|_{\mathcal{M}}
$$

As can be seen by straightforward computation, $\overline{\left\{-\operatorname{div} g \mid g \in K_{1}\right\}}=\overline{B_{1}(0)}$ in $X$, so the supremum on the left-hand side corresponds to the norm of $u$ in $X^{*}$, hence $u$ can be extended to an element in $X^{*}$ (which we do not relabel). Now, $X$ is a closed subspace in $L^{d}\left(\Omega, \operatorname{Sym}^{k}\left(\mathbb{R}^{d}\right)\right)$, so the Hahn-Banach theorem yields an extension $v \in L^{d}\left(\Omega, \operatorname{Sym}^{k}\left(\mathbb{R}^{d}\right)\right)^{*}=$ $L^{d /(d-1)}\left(\Omega, \operatorname{Sym}^{k}\left(\mathbb{R}^{d}\right)\right)$. In particular, $v \in L^{1}\left(\Omega, \operatorname{Sym}^{k}\left(\mathbb{R}^{d}\right)\right)$.

Interpreted as a distribution, $u-v$ belongs to the kernel of $\mathcal{E}$ : For each $g \in \mathcal{C}_{\mathrm{c}}^{\infty}\left(\Omega, \operatorname{Sym}^{k+1}\left(\mathbb{R}^{d}\right)\right)$ we have

$$
\langle u-v,-\operatorname{div} g\rangle=\langle u-u,-\operatorname{div} g\rangle=0
$$

since $-\operatorname{div} g \in X$. Consequently, $u-v$ is a polynomial of degree less than $k$ (see $[5$, Proposition 3.3]) and in particular in $L^{1}\left(\Omega, \operatorname{Sym}^{k}\left(\mathbb{R}^{d}\right)\right)$. Finally, it follows that $u=v+$ $(u-v) \in L^{1}\left(\Omega, \operatorname{Sym}^{k}\left(\mathbb{R}^{d}\right)\right)$, i.e., $u \in \operatorname{BD}\left(\Omega, \operatorname{Sym}^{k}\left(\mathbb{R}^{d}\right)\right)$.

\section{Acknowledgements}

The authors gratefully acknowledge support by the Austrian Science Fund (FWF) special research grant SFB-F32 "Mathematical Optimization and Applications in Biomedical Sciences". 


\section{References}

[1] R. Acar and C. R. Vogel. Analysis of bounded variation penalty methods for ill-posed problems. Inverse Problems, 10(6):1217, 1994.

[2] L. Ambrosio, N. Fusco, and D. Pallara. Functions of Bounded Variation and Free Discontinuity Problems. Oxford Mathematical Monographs. Clarendon Press, 2000.

[3] H. Attouch and H. Brezis. Duality for the sum of convex functions in general Banach spaces. In J. A. Barroso, editor, Aspects of Mathematics and its Applications, volume 34 of North-Holland Mathematical Library, pages 125 - 133. Elsevier, 1986.

[4] J. M. Borwein and J. D. Vanderwerff. Convex Functions: Constructions, Characterizations and Counterexamples. Encyclopedia of Mathematics and its Applications. Cambridge University Press, 2010.

[5] K. Bredies. Symmetric tensor fields of bounded deformation. Annali di Matematica Pura ed Applicata, pages 1-37, 2012.

[6] K. Bredies and M. Holler. Artifact-free decompression and zooming of JPEG compressed images with total generalized variation. In Computer Vision, Imaging and Computer Graphics. Theory and Application, volume 359, pages 242-258. Springer, 2013.

[7] K. Bredies and M. Holler. A TGV regularized wavelet based zooming model. In Scale Space and Variational Methods in Computer Vision, volume 7893, pages 149-160. Springer, 2013.

[8] K. Bredies, K. Kunisch, and T. Pock. Total generalized variation. SIAM Journal on Imaging Sciences, 3(3):492-526, 2010.

[9] K. Bredies, K. Kunisch, and T. Valkonen. Properties of $L^{1}-T G V^{2}$ : The onedimensional case. Journal of Mathematical Analysis and Applications, 398(1):438-454, 2013.

[10] K. Bredies and T. Valkonen. Inverse problems with second-order total generalized variation constraints. In 9th International Conference on Sampling Theory and Applications, Singapore, 2011.

[11] L. C. Evans and R. F. Gariepy. Measure Theory and Fine Properties of Functions. Mathematical Chemistry Series. Taylor \& Francis, 1992.

[12] M. Grasmair. Multi-parameter Tikhonov regularisation in topological spaces. ArXiv preprint 1109.0364, 2011.

[13] B. Hofmann, B. Kaltenbacher, C. Pöschl, and O. Scherzer. A convergence rates result for Tikhonov regularization in Banach spaces with non-smooth operators. Inverse Problems, 23(3):987-1010, 2007.

[14] M. Holler. Higher order regularization for model based data decompression. PhD thesis, University of Graz, 2013. 
[15] K. Ito, B. Jin, and T. Takeuchi. Multi-Parameter Tikhonov Regularization. ArXiv preprint 1102.1173, 2011.

[16] F. Knoll, K. Bredies, T. Pock, and R. Stollberger. Second order total generalized variation (TGV) for MRI. Magnetic Resonance in Medicine, 65(2):480-491, 2011.

[17] F. Knoll, C. Clason, K. Bredies, M. Uecker, and R. Stollberger. Parallel imaging with nonlinear reconstruction using variational penalties. Magnetic Resonance in Medicine, 67(1):34-41, 2012.

[18] F. Knoll, G. Schultz, K. Bredies, D. Gallichan, M. Zaitsev, J. Hennig, and R. Stollberger. Reconstruction of undersampled radial PatLoc imaging using total generalized variation. Magnetic Resonance in Medicine, 70(1):40-52, 2013.

[19] S. Lu and S. V. Pereverzev. Multi-parameter regularization and its numerical realization. Numerische Mathematik, 118:1-31, 2011.

[20] V. Naumova and S. V. Pereverzyev. Multi-penalty regularization with a componentwise penalization. Inverse Problems, 29(7):075002, 2013.

[21] A. Neubauer, T. Hein, B. Hofmann, S. Kindermann, and U. Tautenhahn. Improved and extended results for enhanced convergence rates of Tikhonov regularization in Banach spaces. Applicable Analysis, 89(11):1729-1743, 2010.

[22] C. Pöschl, E. Resmerita, and O. Scherzer. Discretization of variational regularization in Banach spaces. Inverse Problems, 26(10):105017, 2010.

[23] T. Schuster, B. Kaltenbacher, B. Hofmann, and K. S. Kazimierski. Regularization Methods in Banach Spaces. Radon Series on Computational and Applied Mathematics. De Gruyter, 2012.

[24] R. Temam. Mathematical problems in plasticity. Gauthier-Villars, 1985.

[25] R. Temam and G. Strang. Functions of bounded deformation. Archive for Rational Mechanics and Analysis, 75:7-21, 1980.

[26] T. Valkonen, K. Bredies, and F. Knoll. TGV for diffusion tensors: A comparison of fidelity functions. Journal of Inverse and Ill-Posed Problems, 21(3):355-377, 2013.

[27] T. Valkonen, K. Bredies, and F. Knoll. Total generalized variation in diffusion tensor imaging. SIAM Journal on Imaging Sciences, 6(1):487-525, 2013. 